\title{
Reconstruction for the Signature of a Rough Path
}

\author{
Xi Geng*
}

\begin{abstract}
Recently it was proved that the group of rough paths modulo tree-like equivalence is isomorphic to the corresponding signature group through the signature map $S$ (a generalized notion of taking iterated path integrals). However, the proof of this uniqueness result does not contain any information on how to "see" the trajectory of a (tree-reduced) rough path from its signature, and a constructive understanding on the uniqueness result (in particular on the inverse of $S$ ) has become an interesting and important question. The aim of the present paper is to reconstruct a rough path from its signature in an explicit and universal way.
\end{abstract}

MSC 2010 : 34F05(primary), 60G17, 60H10(secondary)

Keywords : Rough paths; Uniqueness of signature; Reconstruction

\section{Introduction}

Motivated from the study of homotopy theory and cohomology of loop spaces, in 1954 K.T. Chen [7] introduced the powerful tool of iterated path integrals. In particular, he observed that a continuous path with bounded variation can be represented by a fully non-commutative power series through an exponential homomorphism. In terms of tensor products, this is equivalent to saying that the integration map $S$, which sends a path $x$ in $\mathbb{R}^{d}$ to the formal tensor series

$$
1+\sum_{n=1}^{\infty} \int_{0<t_{1}<\cdots<t_{n}<1} d x_{t_{1}} \otimes \cdots \otimes d x_{t_{n}}
$$

is a homomorphism from the semigroup of paths under concatenation to the algebra of formal tensor series under tensor product. Moreover, in 1957 Chen [8] discovered an important algebraic property of such representation which asserts that the formal logarithm of $S$ is always a formal Lie series. Equivalently, $S(x)$ satisfies the shuffle product formula for every path $x$.

${ }^{*}$ Department of Mathematical Sciences, Carnegie Mellon University, Pittsburgh, PA 15213, United States. Email: xig@andrew.cmu.edu.

This is the author manuscript accepted for publication and has undergone full peer review but has not been through the copyediting, typesetting, pagination and proofreading process, which may lead to differences between this version and the Version of Record. Please cite this article as doi: 10.1112/plms.12013

This article is protected by copyright. All rights reserved. 
Inspired by the algebraic structure of iterated path integrals, in a seminal work [16] in 1998, T. Lyons developed a theory of path integration and differential equations driven by rough signals, which is now known as rough path theory. The key novel point in his theory is that if a path is irregular, a collection of "generalized iterated integrals" up to a certain degree should be pre-specified in order to define integration against such a path. More precisely, a generic path should be a path taking values in the free nilpotent group up to a certain degree, which is related to the roughness of the underlying path. Identifying the right topology for paths with certain roughness to ensure continuity properties for differential equations is a fundamental contribution in the analytic aspect of rough path theory.

The development of rough path theory leads to tremendous applications in probability theory, due to the important fact that most of interesting continuous stochastic processes can be regarded as rough paths in a canonical way. It follows that a pathwise theory of stochastic differential equations is a consequence of rough path theory. This provides a new perspective in solving a lot of probabilistic problems, for instance regularity of hypoelliptic Gaussian SDEs [6], large deviation principles [13], analysis on path and loop spaces [1] and etc. Rough path theory also provides a one dimensional prototype of M. Hairer's Fields medal work on the theory of regularity structures for stochastic partial differential equations.

An important result in rough path theory, known as Lyons' extension theorem, asserts that a rough path has a unique lifting to the full tensor algebra which has the same regularity. In particular, a rough path can also be represented by a formal tensor series of "generalized iterated integrals" as in the bounded variation case. In the rough path literature, such representation is usually known as the signature of a rough path.

A fundamental question in rough path theory is to understand in what sense is such representation faithful. In 1958, Chen [9] gave an answer to this question for the class of piecewise regular and irreducible paths. After five decades, in 2010 H. Bambly and T. Lyons [12] solved this problem for the class of continuous paths with bounded variation. More precisely, they proved that a continuous path of bounded variation is determined by its signature up to tree-like equivalence. This result was recently extended to the rough path setting by $\mathrm{H}$. Boedihardjo, X. Geng, T. Lyons and D. Yang [4] in 2016.

From a theoretical point of view, the uniqueness result of [4] is important as it builds an isomorphism between the rough path space modulo tree-like equivalence and the corresponding signature group. In particular, it reveals a link between the geometric property of rough paths and the algebraic property of signatures. A natural reason of looking at the signature is that the algebraic structure is very explicit and simple: polynomial functionals on the signature group are always linear functionals. Therefore, regular functions on signature are relatively easy to study. On the practical side, this point plays a key role in the signature approach for time series analysis and financial data analysis (c.f. [14], [17] for instance).

On the other hand, despite of the fact that the signature map $S$ descends to 
an isomorphism between tree-reduced rough paths (a canonical representative in each equivalence class) and their signatures, the proof of the uniqueness result in [4] does not contain any information about how one can "see" the trajectory of a tree-reduced rough path from its signature. The question about reconstructing of a path from its signature is interesting and important as it might shed light on understanding the local geometry of a path from global information (the signature). Moreover, a nice reconstruction method might be useful to understand functions on the rough path space by pulling them back to the signature group through the inverse map $S^{-1}$, and as mentioned before, the resulting functions on signature are generally easier to study. The reconstruction problem was first studied by T. Lyons and W. Xu [18], [19] for the class of piecewise linear paths and the class of continuously differentiable paths in which the modulus of continuity for the derivatives is known.

The aim of the present paper is to study the reconstruction problem in the general rough path setting. To be precise, we aim at reconstructing a treereduced rough path from its signature in an explicit and universal way, in the sense that it relies only on the Euclidean structure where our underlying paths should live, and with the knowledge of any given signature, our reconstruction produces the underlying tree-reduced rough path. The main idea of our reconstruction is motivated from the understanding on Y. Le Jan and Z. Qian's work [15] for Brownian motion into a completely deterministic setting (c.f. Section 3 for a more detailed explanation). We hope that our work might give us a constructive understanding on the uniqueness result in [4], and in particular on the inverse of the signature map.

The present paper is organized in the following way. In Section 2, we recall the basic notions on rough paths and the uniqueness result in [4]. In Section 3, we explain the main idea of our reconstruction, and provide the precise mathematical setting of our problem. In Section 4, we present several preliminary results which are essential for our study. In Section 5, we consider the case when our underlying tree-reduced rough paths are non-self-intersecting. In Section 6,

we deal with the general case. In Section 7 , we give a few concluding remarks on the present work.

\section{Generalities on the Uniqueness Result for the Signature of a Rough Path}

In this section, we present the basic notions on the signature of rough paths and recapture the main result of [4]. We will see that the general idea of understanding non-self-intersecting signature paths in [4] plays an important role in the present work.

Let $T\left(\left(\mathbb{R}^{d}\right)\right)$ be the infinite dimensional tensor algebra over $\mathbb{R}^{d}$ consisting of formal series of tensors in each degree. For $N \in \mathbb{N}$, let

$$
T^{(N)}\left(\mathbb{R}^{d}\right)=\bigoplus_{k=0}^{N}\left(\mathbb{R}^{d}\right)^{\otimes k}
$$

This article is protected by copyright. All rights reserved. 
be the truncated tensor algebra up to degree $N$. Here we identify $\left(\mathbb{R}^{d}\right)^{\otimes k} \cong \mathbb{R}^{d^{k}}$ with basis $\left\{e_{i_{1}} \otimes \cdots \otimes e_{i_{k}}: 1 \leqslant i_{1}, \cdots, i_{k} \leqslant d\right\}$, where $\left\{e_{1}, \cdots, e_{d}\right\}$ is the canonical basis of $\mathbb{R}^{d}$.

Definition 2.1. A multiplicative functional of degree $N \in \mathbb{N}$ is a continuous map $\mathbf{X}_{., .}=\left(1, X_{.,}^{1}, \cdots, X_{.,}^{N}\right)$ from the standard 2-simplex $\Delta=\{(s, t): 0 \leqslant s \leqslant$ $t \leqslant 1\}$ to $T^{(N)}\left(\mathbb{R}^{d}\right)$ satisfying the following so-called Chen's identity:

$$
\mathbf{X}_{s, u}=\mathbf{X}_{s, t} \otimes \mathbf{X}_{t, u}, \forall 0 \leqslant s \leqslant t \leqslant u \leqslant 1 .
$$

Let $\mathbf{X}, \mathbf{Y}$ be two multiplicative functionals of degree $N$. Define

$$
d_{p}(\mathbf{X}, \mathbf{Y})=\max _{1 \leqslant i \leqslant N} \sup _{\mathcal{P}_{[0,1]}}\left(\sum_{l}\left|X_{t_{l-1}, t_{l}}^{i}-Y_{t_{l-1}, t_{l}}^{i}\right|^{\frac{p}{i}}\right)^{\frac{i}{p}},
$$

where $\mathcal{P}_{[0,1]}$ denotes all finite partitions of $[0,1] . d_{p}$ is called the $p$-variation metric, and we say $\mathbf{X}$ has finite (total) $p$-variation if $d_{p}(\mathbf{X}, \mathbf{1})<\infty$ where $\mathbf{1}:=(1,0, \cdots, 0)$. A multiplicative functional $\mathbf{X}$ of degree $\lfloor p\rfloor$ with finite $p$ variation is called a $p$-rough path. The space of $p$-rough paths over $\mathbb{R}^{d}$ is denoted by $\Omega_{p}\left(\mathbb{R}^{d}\right)$.

A fundamental analytic property of $p$-rough paths is the following extension theorem proved by Lyons [16]. It asserts that some analytic version of "iterated path integrals" against a $p$-rough path can be uniquely defined and has a nice factorial decay property.

Theorem 2.1. (Lyons' extension theorem) Let $\mathbf{X}$ be a p-rough path. Then for any $i \geqslant\lfloor p\rfloor+1$, there exists a unique continuous map $X^{i}: \Delta \rightarrow\left(\mathbb{R}^{d}\right)^{\otimes i}$ such that

$$
S(\mathbf{X}):=\left(1, X^{1}, \cdots, X^{\lfloor p\rfloor}, \cdots X^{i}, \cdots\right)
$$

is a multiplicative functional in $T\left(\left(\mathbb{R}^{d}\right)\right)$ with finite $p$-variation when restricted up to each degree. Moreover, there exists a positive constant $C_{p}$ depending only on $p$, such that

$$
\left|X_{s, t}^{i}\right| \leqslant \frac{C_{p} \omega(s, t)^{\frac{i}{p}}}{\left(\frac{i}{p}\right) !}, \forall i \geqslant 1 \text { and } \forall(s, t) \in \Delta,
$$

where $\omega$ is the control function defined by

$$
\omega(s, t)=\sum_{i=1}^{\lfloor p\rfloor} \sup _{\mathcal{P}_{[s, t]}} \sum_{l}\left|X_{t_{l-1}, t_{l}}^{i}\right|^{\frac{p}{i}},(s, t) \in \Delta .
$$

Definition 2.2. The tensor element $S(\mathbf{X})_{0,1} \in T\left(\left(\mathbb{R}^{d}\right)\right)$ defined by Theorem 2.1 is called the signature of $\mathbf{X}$.

This article is protected by copyright. All rights reserved. 
In the case of $p=1$, a $p$-rough path $\mathbf{X}$ is just the collection of increments

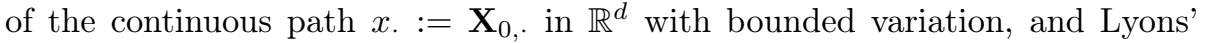
extension of $\mathbf{X}$ reduces to the classical iterated path integrals along $x$.

Now let $G\left(\mathbb{R}^{d}\right)$ be the group defined by the exponential of formal Lie series in $T\left(\left(\mathbb{R}^{d}\right)\right)$. For $N \in \mathbb{N}$, let $G^{N}\left(\mathbb{R}^{d}\right)$ be the truncation of $G\left(\mathbb{R}^{d}\right)$ up to degree $N$, which consists of the exponential of Lie polynomials up to degree $N$ in $T^{(N)}\left(\mathbb{R}^{d}\right)$. $G^{N}\left(\mathbb{R}^{d}\right)$ is usually known as the free nilpotent group of degree $N$.

The following result, known as the shuffle product formula, reveals a fundamental algebraic property for the signature of continuous paths with bounded variation (c.f. Reutenauer [21] and also Chen [8]). This property lies as a crucial base to expect that a path with bounded variation is uniquely determined by its signature up to tree-like equivalence and also to its extension to the rough path setting.

Theorem 2.2. (Shuffle product formula) A tensor element $a=\left(1, a^{1}, a^{2}, \cdots\right) \in$ $T\left(\left(\mathbb{R}^{d}\right)\right)$ belongs to $G\left(\mathbb{R}^{d}\right)$ if and only if

$$
a^{m} \otimes a^{n}=\sum_{\sigma \in \mathcal{S}(m, n)} \mathcal{P}^{\sigma}\left(a^{m+n}\right), \forall m, n \geqslant 1,
$$

where $\mathcal{S}(m, n)$ denotes the set of $(m, n)$-shuffles in the permutation group of order $m+n$, and $\mathcal{P}^{\sigma}: V^{\otimes(m+n)} \rightarrow V^{\otimes(m+n)}$ is the permutation operator given by

$$
\mathcal{P}^{\sigma}\left(v_{1} \otimes \cdots \otimes v_{m+n}\right)=v_{\sigma(1)} \otimes \cdots \otimes v_{\sigma(m+n)} .
$$

In particular, for any continuous path $x$ in $\mathbb{R}^{d}$ with bounded variation, the signature of $x$ satisfies (2.2) and hence belongs to $G\left(\mathbb{R}^{d}\right)$.

Following the general setting of [4], in the present paper we work with a class of rough paths called weakly geometric rough paths. It is defined on the previous algebraic structure and is the fundamental class of paths that the theory of rough integration and differential equations is based on.

Definition 2.3. A weakly geometric p-rough path is a p-rough path taking values in $G^{\lfloor p\rfloor}\left(\mathbb{R}^{d}\right)$. The space of weakly geometric $p$-rough paths over $\mathbb{R}^{d}$ is denoted by $W G \Omega_{p}\left(\mathbb{R}^{d}\right)$.

It can be shown (c.f. [10], and also [4]) that the signature of a weakly geometric rough path is an element in $G$.

Remark 2.1. There is an equivalent intrinsic definition of weakly geometric rough paths in terms of the Carnot-Carathéodory metric $d_{C C}$ on $G^{\lfloor p\rfloor}\left(\mathbb{R}^{d}\right)$. To be precise, a weakly geometric $p$-rough path is a continuous path $\mathbf{X}:[0,1] \rightarrow$ $G^{\lfloor p\rfloor}\left(\mathbb{R}^{d}\right)$ starting at the unit such that $\mathbf{X}$ has finite $p$-variation with respect to the metric $d_{C C}$. This definition is equivalent to Definition 2.3 through $\mathbf{X}_{s, t}=$ $X_{s}^{-1} \otimes \mathbf{X}_{t}$ and $\mathbf{X}_{t}=\mathbf{X}_{0, t}$. We refer the reader to [10] for a detailed discussion along this approach.

This article is protected by copyright. All rights reserved. 
From now on, unless otherwise stated we always regard a weakly geometric $p$-rough path as an actual path in $G^{\lfloor p\rfloor}\left(\mathbb{R}^{d}\right)$ instead of a multiplicative functional defined on the simplex $\Delta$.

The main result of [4] states that a weakly geometric $p$-rough path is uniquely determined by its signature up to tree-like equivalence. More precisely, it was shown that:

Theorem 2.3. A weakly geometric p-rough path $\mathbf{X}$ has trivial signature if and only if it is tree-like, in the sense that there exists some real tree $\tau$ together with a continuous loop $\alpha:[0,1] \rightarrow \tau$ and some continuous map $\psi: \tau \rightarrow W G \Omega_{p}\left(\mathbb{R}^{d}\right)$ such that $\mathbf{X}=\psi \circ \alpha$.

In particular, let

$$
\mathfrak{S}_{p}=\left\{g=S(\mathbf{X})_{0,1}: \mathbf{X} \in W G \Omega_{p}\left(\mathbb{R}^{d}\right)\right\}
$$

be the signature group for weakly geometric $p$-rough paths. It was proved that for each $g \in \mathfrak{S}_{p}$, there exists a unique $\mathbf{X}^{g} \in W G \Omega_{p}\left(\mathbb{R}^{d}\right)$ up to reparametrization, such that $S\left(\mathbf{X}^{g}\right)_{0,1}=g$ and $S\left(\mathbf{X}^{g}\right)_{0}$, is a non-self-intersecting (or simple) path in $T\left(\left(\mathbb{R}^{d}\right)\right)$. $\mathbf{X}^{g}$ is known as the tree-reduced path associated with signature $g$. It follows that $\mathfrak{S}_{p}$ can be equipped with a real tree metric in a canonical way, and a weakly geometric $p$-rough path with trivial signature factors through this real tree with $\psi$ being the projection map.

Therefore, modulo tree-like paths the signature homomorphism

$$
\begin{aligned}
S_{p}: W G \Omega_{p}\left(\mathbb{R}^{d}\right) & \rightarrow T\left(\left(\mathbb{R}^{d}\right)\right), \\
\mathbf{X} & \mapsto S(\mathbf{X})_{0,1},
\end{aligned}
$$

descents to a group isomorphism

$$
\widetilde{S}_{p}: W G \Omega_{p}\left(\mathbb{R}^{d}\right) / \text { tree-like } \stackrel{\cong}{\rightrightarrows} \mathfrak{S}_{p} .
$$

Moreover, each equivalence class contains a unique tree-reduced path $\mathbf{X}$ (up to reparametrization) and this isomorphism gives rise to a one-to-one correspondence between tree-reduced paths (up to reparametrization) and signatures.

However, the proof of Theorem 2.3 contains no information about how a tree-reduced path can be reconstructed from its signature. The development of such a reconstruction in an explicit and universal way is the main focus of the present paper.

Before studying the reconstruction problem, let us mention the following interesting fact. In the case of $p=1$, it is not hard to see that (c.f. [12]) a path is tree-reduced if and only if it is a reparametrization of the unique minimizer of 1-variation (i.e. the length) among paths parametrized by arc length with the same signature. However, this is not true in the case of $p>1$. A tree-reduced path certainly minimizes the $p$-variation, but a $p$-variation minimizer might not be tree-reduced no matter how it is parametrized. We conclude this section by providing such an example.

This article is protected by copyright. All rights reserved. 
Example 2.1. Consider the two dimensional case and $1<p<2$. Let $\overparen{A B}$ be an arc of the unit circle centered at $O \in \mathbb{R}^{2}$ with central angle $\theta_{0}$, and let $C$ be the midpoint of $\widehat{A B}$. Let $D$ be a point on the extension of the radius vector $\overrightarrow{O C}$ and let $|C D|=\varepsilon$. Consider the paths $x, y:[0,1] \rightarrow \mathbb{R}^{2}$ defined by the trajectories

$$
x=\overparen{A C} \sqcup \overrightarrow{C D} \sqcup \overrightarrow{D C} \sqcup \overparen{C B}, y=\overparen{A B},
$$

respectively, where " $\sqcup$ " means concatenation. It is easy to see that $x, y$ have the same signature $g$ and $y$ is a tree-reduced path. Apparently $x$ and $y$ do not differ by just a reparametrization. Now we show that $\|x\|_{p-\text { var }}=\|y\|_{p-\text { var }}=|\overrightarrow{A B}|$ provided $\theta_{0}$ and $\varepsilon$ are small enough.

In fact, let $E \in \overparen{A B}$ and denote the central angle $\angle E O B$ by $\theta$. Consider the function

$$
f(\theta)=|\overrightarrow{A E}|^{p}+|\overrightarrow{E B}|^{p}, \theta \in\left[0, \theta_{0}\right],
$$

which can be written as

$$
f(\theta)=2^{p}\left(\sin ^{p} \frac{\theta}{2}+\sin ^{p} \frac{\theta_{0}-\theta}{2}\right)
$$

according to Euclidean geometry. Computing the second derivative of $f$, we obtain that

$$
f^{\prime \prime}(\theta)=\frac{p}{2^{2-p}}\left((p-1)\left(\frac{\cos ^{2} \frac{\theta}{2}}{\sin ^{2-p} \frac{\theta}{2}}+\frac{\cos ^{2} \frac{\theta_{0}-\theta}{2}}{\sin ^{2-p} \frac{\theta_{0}-\theta}{2}}\right)-\left(\sin ^{p} \frac{\theta}{2}+\sin ^{p} \frac{\theta_{0}-\theta}{2}\right)\right) .
$$

Since $1<p<2$, we know that when $\theta_{0}$ is small, $f^{\prime \prime}(\theta)$ is uniformly positive and hence $f$ is convex on $[0, \theta]$. Also note that $f(0)=f\left(\theta_{0}\right)=|\overrightarrow{A B}|^{p}$. Therefore, for $\theta_{0}$ small enough $f$ obtains its maximum on the end points and we have

$$
|\overrightarrow{A E}|^{p}+|\overrightarrow{E B}|^{p} \leqslant|\overrightarrow{A B}|^{p}, \forall E \in \overparen{A B} .
$$

Now we fix such $\theta_{0}$. This already implies that $\|y\|_{p}=|\overrightarrow{A B}|$. Moreover, by considering the symmetry of $f(\theta)$ it is easy to see that $f$ obtains its minimum at $\theta=\theta_{0} / 2$. Set

$$
\lambda=|\overrightarrow{A B}|^{p}-|\overrightarrow{A C}|^{p}-|\overrightarrow{C B}|^{p}>0 .
$$

It remains to show that when $\varepsilon$ is small enough, $\|x\|_{p}=|\overrightarrow{A B}|$. To this end, let

$$
\mathcal{P}: 0=t_{0}<t_{1}<\cdots<t_{n}=1
$$

be a finite partition of $[0,1]$, and let $t_{k}, t_{l}$ be the first and last partition points at which $x$ is in $\overline{C D}$ respectively. If such points do not exist, then obviously we have

$$
\sum_{i=1}^{n}\left|x_{t_{i}}-x_{t_{i-1}}\right|^{p} \leqslant|\overrightarrow{A B}|^{p}
$$

This article is protected by copyright. All rights reserved. 
Otherwise, we have

$$
\begin{aligned}
\sum_{i=1}^{n}\left|x_{t_{i}}-x_{t_{i-1}}\right|^{p}= & \sum_{i=1}^{k-1}\left|x_{t_{i}}-x_{t_{i-1}}\right|^{p}+\left|x_{t_{k}}-x_{t_{k-1}}\right|^{p}+\sum_{i=k+1}^{l}\left|x_{t_{i}}-x_{t_{i-1}}\right|^{p} \\
& +\left|x_{t_{l+1}}-x_{t_{l}}\right|^{p}+\sum_{i=l+2}^{n}\left|x_{t_{i}}-x_{t_{i-1}}\right|^{p} \\
\leqslant & \left|x_{t_{k-1}}-A\right|^{p}+\left|B-x_{t_{l+1}}\right|^{p}+\left|x_{t_{k}}-x_{t_{k-1}}\right|^{p} \\
& +\left|x_{t_{l+1}}-x_{t_{l}}\right|^{p}+2 \varepsilon^{p}
\end{aligned}
$$

where we have used the previous discussion and the fact that $\overrightarrow{C D}$ is a geodesic. It follows that

$$
\begin{aligned}
& \sum_{i=1}^{n}\left|x_{t_{i}}-x_{t_{i-1}}\right|^{p} \\
\leqslant & |\overrightarrow{A C}|^{p}+|\overrightarrow{C B}|^{p}+\left(\left|x_{t_{k}}-x_{t_{k-1}}\right|^{p}-\left|C-x_{t_{k-1}}\right|^{p}\right) \\
& +\left(\left|x_{t_{l+1}}-x_{t_{l}}\right|^{p}-\left|x_{t_{l+1}}-C\right|^{p}\right)+2 \varepsilon^{p} \\
= & |\overrightarrow{A B}|^{p}-\left(|\overrightarrow{A B}|^{p}-|\overrightarrow{A C}|^{p}-|\overrightarrow{C B}|^{p}-\left(\left|x_{t_{k}}-x_{t_{k-1}}\right|^{p}-\left|C-x_{t_{k-1}}\right|^{p}\right)\right. \\
& \left.-\left(\left|x_{t_{l+1}}-x_{t_{l}}\right|^{p}-\left|x_{t_{l+1}}-C\right|^{p}\right)-2 \varepsilon^{p}\right) .
\end{aligned}
$$

On the other hand, we have

$$
\begin{aligned}
\left|x_{t_{k}}-x_{t_{k-1}}\right|^{p}-\left|C-x_{t_{k-1}}\right|^{p} & \leqslant\left(\left|C-x_{t_{k-1}}\right|+\varepsilon\right)^{p}-\left|C-x_{t_{k-1}}\right|^{p} \\
& \leqslant \max \left\{(\sqrt{\varepsilon}+\varepsilon)^{p}, \theta_{0}^{p}\left((1+\sqrt{\varepsilon})^{p}-1\right)\right\} \\
& =: \mu(\varepsilon) .
\end{aligned}
$$

The same inequality holds for $\left|x_{t_{l+1}}-x_{t_{l}}\right|^{p}-\left|x_{t_{l+1}}-C\right|^{p}$. Therefore, we have

$$
\sum_{i=1}^{n}\left|x_{t_{i}}-x_{t_{i-1}}\right|^{p} \leqslant|\overrightarrow{A B}|^{p}-\left(\lambda-2 \mu(\varepsilon)-2 \varepsilon^{p}\right) \leqslant|\overrightarrow{A B}|^{p}
$$

provided $\varepsilon$ is small enough so that

$$
2 \mu(\varepsilon)+2 \varepsilon^{p}<\lambda .
$$

Now by taking supremum over all finite partitions of $[0,1]$, we conclude that

$$
\|x\|_{p} \leqslant|\overrightarrow{A B}|=\|y\|_{p} \leqslant\|x\|_{p} .
$$

Therefore, $x$ is also a $p$-variation minimizer with signature $g$. 


\section{Main Idea of the Reconstruction}

In this section, we explain the basic idea of our reconstruction and present the precise mathematical setting of our problem.

The underlying strategy of our reconstruction is motivated from the probabilistic work of Le Jan and Qian [15] for Brownian motion, which was further developed by X. Geng Z. Qian [11] for hypoelliptic diffusions and by H. Boedihardjo and X. Geng [3] in the non-Markov setting. We first summarize the original idea of Le Jan and Qian for the almost-sure reconstruction of Brownian sample paths.

(1) By knowing the (Stratonovich) signature of Brownian sample paths, the shuffle product formula allows us to construct iterated path integrals along any finite sequence of regular one forms (which they called extended signatures).

(2) Given a disjoint family of nice compact domains $\left\{K_{n}\right\}$ in space with a well-chosen one form supported in each $K_{n}$, by evaluating extended signatures associated with this family of one forms, with probability one we can determine the ordered sequence of domains $K_{n}$ visited by a Brownian path.

(3) Construct a polygonal path associated with this ordered sequence of domains $K_{n}$ visited by the underlying Brownian path. As we refine the geometric scheme, it is reasonable to expect that the polygonal approximation converges to the original Brownian path in some sense.

In the probabilistic setting, the way of choosing the "testing" one forms and of refining the geometric scheme to obtain convergence depends on the a priori knowledge on the law of the underlying process, in particular on its certain nondegeneracy properties. In this respect, it gives rise to the main obstruction of developing such idea in the deterministic setting for arbitrary weakly geometric rough paths as we need to treat every path equally. We explain this point in more details.

For simplicity, let us consider the two dimensional case in which our underlying paths are non-self-intersecting and have bounded variation. Assume that the plane is decomposed into disjoint squares of size order $\varepsilon$ and narrow tunnels of width order $\delta(\delta<<\varepsilon)$. By using extended signatures we can determine the ordered sequence $\mathbf{m}^{\varepsilon, \delta}$ of squares visited by a path (this relies on the non-selfintersecting assumption on our path in a crucial way), and we can construct a polygonal path associated with this sequence according to (3) as discussed before.

Now if we refine our geometric scheme by letting $(\varepsilon, \delta) \rightarrow 0$ independently, we cannot expect that the polygonal approximation would converge to the original path in any sense although it should be true generically. In general, for any given refinement of geometric schemes, one could always construct a path such that the convergence fails for this path. Heuristically, such a path should have

a long excursion in the complement of each geometric scheme (i.e. the tunnels in this case) along the refinement.

The following is a simple example illustrating this situation.

Example 3.1. Figure 3.1 gives a simple example that the convergence of the

This article is protected by copyright. All rights reserved. 


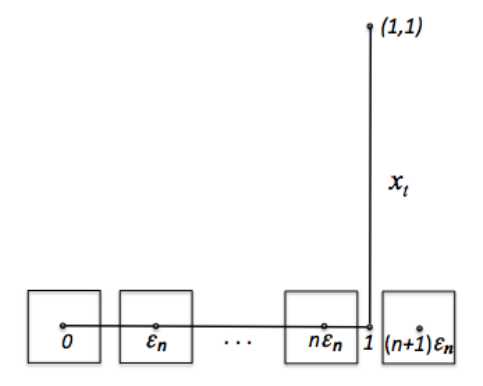

Figure 3.1: An example illustrating that the convergence of polygonal approximation can fail as $(\varepsilon, \delta) \rightarrow 0$.

polygonal approximation could fail in general. Here $x$ is a piecewise linear path starting from origin with two edges, going first from $(0,0)$ to $(1,0)$ and then from $(1,0)$ to $(1,1)$. In the $\varepsilon$-scale, every square is centered at $\varepsilon \boldsymbol{n}$ for some integer point $\boldsymbol{n}$ in the plane, leaving gaps between squares to the order of another independent parameter $\delta$. Now choose a subsequence $\varepsilon_{n}=\frac{2}{2 n+1}$ and arbitrary $\delta_{n}$. It is then easy to see that along this subsequence $\varepsilon_{n}$, the polygonal approximation $x^{n}$ is given by the linear path joining the origin and the point $n \varepsilon_{n}$. In this situation we cannot expect that $x^{n}$ converges to the original path $x$ in any reasonable sense.

In a more general situation, suppose that $\left\{\mathcal{C}_{n}\right\}$ is any given refinement of geometric schemes in $\mathbb{R}^{d}$ (i.e. the size of domains goes to zero uniformly as $n \rightarrow \infty)$, and let $0<r<R$. Then one can prove that there exists a subsequence $\mathcal{C}_{l_{n}}$ and a continuous path $x:[0,1] \rightarrow \mathbb{R}^{d}$, such that $\left|x_{1}\right| \geqslant R$ but the polygonal approximation $x^{n}$ of $x$ with respect to $\mathcal{C}_{l_{n}}$ is contained in $B(0, r)$ for all sufficiently large $n$. Therefore, one could not expect that $x^{n}$ converges to $x$ in any reasonable sense. To get an idea of how this works, we can first pick some geometric scheme $\mathcal{C}_{l_{1}}$ and construct a path $x^{1}$ with $\left|x_{1}^{1}\right| \geqslant R$ but which does not enter domains in $\mathcal{C}_{l_{1}}$ which are outside $B(0, r)$. Then we can pick some next geometric scheme $\mathcal{C}_{l_{2}}$ and modify $x^{1}$ to another path $x^{2}$ with $\left|x_{1}^{2}\right| \geqslant R$ but which does not enter domains in $\mathcal{C}_{l_{1}}$ and $\mathcal{C}_{l_{2}}$ which are outside $B(0, r)$. By doing this inductively and keeping track of the uniform error created in each step, we can obtain a sequence of paths $x^{n}$ which converges uniformly to some limit path $x$ with $\left|x_{1}\right| \geqslant R$, and $x^{n}$ does not enter domains in $\mathcal{C}_{l_{1}}, \cdots, \mathcal{C}_{l_{n}}$ which are outside $B(0, r)$. It is then natural to expect that the limit path $x$ does not enter domains in $\mathcal{C}_{l_{n}}$ which are outside $B(0, r)$ for every $n$. Therefore, $x$ will be a desired counter-example. The detailed proof of this claim is quite technical and is hence omitted.

The main idea of overcoming this issue is to let the signature $g$ determine the width $\delta$ of the tunnels for each $\varepsilon$ according to some stable quantity, so that the underlying path with signature $g$ is not able to travel through any long narrow tunnels in the resulting geometric scheme. Therefore the approximating polyg-

This article is protected by copyright. All rights reserved. 
onal path must converge to the original path. This part is the key ingredient of the present work.

If the underlying path is not simple, the situation is more complicated since extended signatures might not be able to recover the discrete route $\mathbf{m}^{\varepsilon, \delta}$. The key to overcoming this issue is to lift the path to its truncated signature path up to certain high degree $N$ which is determined by the signature $g$. This is achieved again through some stabilizing property. Here we will see that the decay of signature plays a crucial role.

Now we present the basic functional setting on which our reconstruction is based. Throughout the rest of the present paper, the roughness $p \geqslant 1$ is fixed.

As the signature is invariant under reparametrization, it is natural to regard the parametrization-free tree-reduced path as a single object to be reconstructed in some functional space.

Definition 3.1. A reparametrization is a continuous and strictly increasing function $\sigma:[0,1] \rightarrow[0,1]$, such that $\sigma(0)=0$ and $\sigma(1)=1$. The group of reparametrizations is denoted by $\mathcal{R}$.

Let $(E, \rho)$ be a metric space, and $W=C([0,1] ; E)$ be the space of continuous paths in $E$. We introduce an equivalence relation " $\sim$ " on $W$ by $x \sim x^{\prime}$ if and only if $x .=x_{\sigma(\cdot)}^{\prime}$ for some $\sigma \in \mathcal{R}$, and let $W^{\sim}$ be the corresponding quotient space. Now define a distance function $d$ on $W^{\sim}$ by

$$
d\left([x],\left[x^{\prime}\right]\right)=\inf _{\sigma \in \mathcal{R}} \sup _{t \in[0,1]} \rho\left(x_{t}, x_{\sigma(t)}^{\prime}\right),[x],\left[x^{\prime}\right] \in W^{\sim} .
$$

It is not hard to see that $d$ is well-defined, symmetric and satisfies the triangle inequality (c.f. [3] for a detailed discussion). However, it is not positively definite in general. Now let $\Gamma$ be the set of paths $x \in W$ such that there exist $0 \leqslant s<t \leqslant 1$ with $x_{u}=x_{s}$ for all $u \in[s, t]$. If we restrict the relation " $\sim$ " on $W_{0}=\Gamma^{c} \subset W$ and consider the corresponding quotient space $W_{0}^{\sim}$, it is proved in [3] that $d$ is indeed a metric on $W_{0}^{\sim}$.

In our situation, we take $E$ to be the space $E_{\lfloor p\rfloor}=T^{\lfloor p\rfloor}\left(\mathbb{R}^{d}\right)$. Let $\mathbf{X}$ be a weakly geometric $p$-rough path. If $\mathbf{X}$ stays constant during some $[s, t]$, then its signature path would not be simple. It follows that every tree-reduced weakly geometric $p$-rough path can be regarded as an element in $W_{0}$ (recall that $G^{\lfloor p\rfloor}\left(\mathbb{R}^{d}\right)$ is canonically embedded in $\left.E_{\lfloor p\rfloor}\right)$. Moreover, from the uniqueness result we know that any two tree-reduced path with the same signature differ by a reparametrization in the sense of Definition 3.1. Therefore, the set of tree-reduced paths modulo reparametrization can naturally be regarded as a subset $\mathcal{T}_{p}$ of $W_{0}^{\sim}$. Our aim is to reconstruct the unique element in $\mathcal{T}_{p}$ corresponding to each given $g \in \mathfrak{S}_{p}$. 


\section{Extended Signatures for Truncated Signature Paths}

The starting point of our reconstruction is the use of extended signatures which are iterated integrals along ordered sequence of one forms. In this section we present the basic ingredients for this part.

\subsection{The Signature of Truncated Signature Paths}

Since we aim at reconstructing the tree-reduced path $\mathbf{X}$ as a trajectory in $E_{\lfloor p\rfloor}$, we shall integrate along one forms defined on $E_{\lfloor p\rfloor}$ rather than those on $\mathbb{R}^{d}$. This requires an important fact that $\mathbf{X}$ can be regarded as the first level path of some weakly geometric $p$-rough path $\mathbf{Y}$ over $E_{\lfloor p\rfloor}$ whose signature, which is an element of the tensor algebra over $E_{\lfloor p\rfloor}$, is determined by the signature of $\mathbf{X}$ explicitly. Fortunately, such $\mathbf{Y}$ can be constructed in a canonical way. A construction based on ordered shuffles already appears in [5], [16] for the study of rough integration against weakly geometric rough paths, and also in [4] for the uniqueness problem. Here for completeness we present an equivalent construction which does not rely on ordered shuffles and is more convenient to use from a combinatorial point of view.

Let $\mathbf{X}$ be an arbitrary weakly geometric $p$-rough path.

Given $N \in \mathbb{N}$, let $y_{t}=\left(1, X_{0, t}^{1}, \cdots, X_{0, t}^{N}\right)$ be the truncated signature path of $\mathbf{X}$ up to degree $N$. Then we know that $y$ takes values in the Euclidean space

$$
E_{N}:=\bigoplus_{i=0}^{N}\left(\mathbb{R}^{d}\right)^{\otimes i}
$$

Therefore, the construction of the lifting of $y$ should take place in the tensor algebra over $E_{N}$, where for each $n \geqslant 1$, we apply the following identification:

$$
\left(E_{N}\right)^{\otimes n} \cong \bigoplus_{i_{1}, \cdots, i_{n}=0}^{N}\left(\mathbb{R}^{d}\right)^{\otimes\left(i_{1}+\cdots+i_{n}\right)} .
$$

Equivalently we need to construct $Y_{s, t}^{n ; i_{1}, \cdots, i_{n}} \in\left(\mathbb{R}^{d}\right)^{\otimes\left(i_{1}+\cdots+i_{n}\right)}$ for $n \geqslant 1,0 \leqslant$ $i_{1}, \cdots, i_{n} \leqslant N$ and $(s, t) \in \Delta$. $Y^{1}$ is just the increment of $y$.

Now we consider the case when $n=2$. We first proceed by a formal calculation to motivate the rigorous construction. Such calculation is based on the formal differential equation

$$
d S(\mathbf{X})_{s, t}=S(\mathbf{X})_{s, t} \otimes d x_{t}
$$

for the signature path (c.f. [10], Proposition 7.8) together with the shuffle product formula. Note that the identity in each step below do not make sense when $p \geqslant 2$.

This article is protected by copyright. All rights reserved. 
If $1 \leqslant i_{1}, i_{2} \leqslant N$, then formally we have

$$
\begin{aligned}
& Y_{s, t}^{2 ; i_{1}, i_{2}} \\
& \doteq \int_{s<u<t} Y_{s, u}^{1 ; i_{1}} \otimes d y_{u}^{i_{2}} \\
& \doteq \int_{s<u<t}\left(X_{0, u}^{i_{1}}-X_{0, s}^{i_{1}}\right) \otimes d X_{0, u}^{i_{2}} \\
& \doteq \int_{s<u<t}\left(\sum_{j_{1}=1}^{i_{1}} X_{0, s}^{i_{1}-j_{1}} \otimes X_{s, u}^{j_{1}}\right) X_{0, u}^{i_{2}-1} \otimes d x_{u} \\
& \doteq \int_{s<u<t}\left(\sum_{j_{1}=1}^{i_{1}} X_{0, s}^{i_{1}-j_{1}} \otimes X_{s, u}^{j_{1}}\right)\left(\sum_{j_{2}=0}^{i_{2}-1} X_{0, s}^{i_{2}-1-j_{2}} \otimes X_{s, u}^{j_{2}}\right) \otimes d x_{u} \\
& \doteq \sum_{j_{1}=1}^{i_{1}} \sum_{j_{2}=0}^{i_{2}-1} \int_{s<u<t} P^{\tau\left(i_{1}-j_{1}, j_{1}, i_{2}-1-j_{2}, j_{2}\right)}\left(X_{0, s}^{i_{1}-j_{1}} \otimes X_{0, s}^{i_{2}-1-j_{2}}\right. \\
& \left.\otimes X_{s, u}^{j_{1}} \otimes X_{s, u}^{j_{2}}\right) \otimes d x_{u} \\
& \doteq \sum_{j_{1}=1}^{i_{1}} \sum_{j_{2}=0}^{i_{2}-1} \int_{s<u<t} P^{\tau\left(i_{1}-j_{1}, i_{1}, i_{2}-1-j_{2}, j_{2}\right)} \\
& \left(\sum_{\substack{\sigma_{1} \in \mathcal{S}\left(i_{1}-j_{1}, i_{2}-1-j_{2}\right) \\
\sigma_{2} \in \mathcal{S}\left(j_{1}, j_{2}\right)}} P^{\sigma_{1} \otimes \sigma_{2}}\left(X_{0, s}^{i_{1}+i_{2}-j_{1}-j_{2}-1} \otimes X_{s, u}^{j_{1}+j_{2}}\right)\right) \otimes d x_{u} \\
& \doteq \sum_{j_{1}=1}^{i_{1}} \sum_{j_{2}=0}^{i_{2}-1} \sum_{\substack{\sigma_{1} \in \mathcal{S}\left(i_{1}-j_{1}, i_{2}-1-j_{2}\right) \\
\sigma_{2} \in \mathcal{S}\left(j_{1}, j_{2}\right)}} \bar{P}^{\tau\left(i_{1}-j_{1}, i_{1}, i_{2}-1-j_{2}, i_{2}\right)} \\
& \circ \bar{P}^{\sigma_{1} \otimes \sigma_{2}}\left(X_{0, s}^{i_{1}+i_{2}-j_{1}-j_{2}-1} \otimes X_{s, u}^{j_{1}+j_{2}+1}\right) .
\end{aligned}
$$

Here $P^{\tau\left(i_{1}-j_{1}, j_{1}, i_{2}-1-j_{2}, j_{2}\right)}, P^{\sigma_{1} \otimes \sigma_{2}}$ are linear transformations on $\left(\mathbb{R}^{d}\right)^{\otimes\left(i_{1}+i_{2}-1\right)}$ defined by

$$
P^{\tau\left(i_{1}-j_{1}, j_{1}, i_{2}-1, j_{2}\right)}(a \otimes b \otimes c \otimes d)=a \otimes c \otimes b \otimes d
$$

for $a \in\left(\mathbb{R}^{d}\right)^{\otimes\left(i_{1}-j_{1}\right)}, b \in\left(\mathbb{R}^{d}\right)^{\otimes\left(i_{2}-1-j_{2}\right)}, c \in\left(\mathbb{R}^{d}\right)^{\otimes j_{1}}, d \in\left(\mathbb{R}^{d}\right)^{\otimes j_{2}}$, and

$$
P^{\sigma_{1} \otimes \sigma_{2}}(a \otimes b)=P^{\sigma_{1}}(a) \otimes P^{\sigma_{2}}(b)
$$

for $a \in\left(\mathbb{R}^{d}\right)^{\otimes\left(i_{1}+i_{2}-j_{1}-j_{2}-1\right)}, b \in\left(\mathbb{R}^{d}\right)^{\otimes\left(j_{1}+j_{2}\right)} . \bar{P}^{\tau\left(i_{1}-j_{1}, i_{1}, i_{2}-1-j_{2}, i_{2}\right)}, \bar{P}^{\sigma_{1} \otimes \sigma_{2}}$ are their extensions to $\left(\mathbb{R}^{d}\right)^{\left(i_{1}+i_{2}\right)}$ by fixing the last tensor component. The notation "ㄹ" means that the identities are formal. For simplicity, we can write (4.1) as

$$
Y_{s, t}^{2 ; i_{1}, i_{2}} \doteq \sum_{j=2}^{i_{1}+i_{2}} \sum_{\sigma \in \mathcal{A}\left(i_{1}, i_{2}, j\right)} P^{\sigma}\left(X_{0, s}^{i_{1}+i_{2}-j} \otimes X_{s, t}^{j}\right)
$$

This article is protected by copyright. All rights reserved. 
where $\mathcal{A}\left(i_{1}, i_{2}, j\right)$ is a set of permutations of order $i_{1}+i_{2}$ which fix the last component. Of course $\mathcal{A}\left(i_{1}, i_{2}, j\right)$ can be written down explicitly from (4.1) but we are not interested in its exact expression. The crucial point here is that the right hand side of (4.2) is a well-defined element in $\left(\mathbb{R}^{d}\right)^{\otimes\left(i_{1}+i_{2}\right)}$, and it is reasonable to take it as the definition of $Y_{s, t}^{2 ; i_{1}, i_{2}}$.

Inductively, assume that we have defined

$$
Y_{s, t}^{n ; i_{1}, \cdots, i_{n}}=\sum_{j=n}^{i_{1}+\cdots+i_{n}} \sum_{\sigma \in \mathcal{A}\left(i_{1}, \cdots, i_{n}, j\right)} P^{\sigma}\left(X_{0, s}^{i_{1}+\cdots+i_{n}-j} \otimes X_{s, t}^{j}\right)
$$

for all $1 \leqslant i_{1}, \cdots, i_{n} \leqslant N$ and $(s, t) \in \Delta$, where $\mathcal{A}\left(i_{1}, \cdots, i_{n}, j\right)$ is a set of permutations of order $i_{1}+\cdots+i_{n}$ which fix the last component. Then for $1 \leqslant i_{1}, \cdots, i_{n+1} \leqslant N$, formally we have

$$
\begin{aligned}
& \int_{s<u<t}^{Y_{s, t}^{n+1 ; i_{1}, \cdots, i_{n+1}}} Y_{s, u}^{n ; i_{1}, \cdots, i_{n}} \otimes d y_{u}^{i_{n+1}} \\
= & \sum_{j=n}^{i_{1}+\cdots+i_{n}} \sum_{\sigma \in \mathcal{A}\left(i_{1}, \cdots, i_{n}, j\right)} \int_{s<u<t} \bar{P}^{\sigma}\left(X_{0, s}^{i_{1}+\cdots+i_{n}-j} \otimes X_{s, u}^{j} \otimes X_{0, u}^{i_{n+1}-1}\right) \otimes d x_{u},
\end{aligned}
$$

where $\bar{P}^{\sigma}$ is the extension of $P^{\sigma}$ to $\left(\mathbb{R}^{d}\right)^{\otimes\left(i_{1}+\cdots+i_{n+1}-1\right)}$ by fixing the last $i_{n+1}-1$ tensor components. By applying the same formal calculation as in the case when $n=2$, we obtain that

$$
Y_{s, t}^{n+1 ; i_{1}, \cdots, i_{n}, i_{n+1}} \doteq \sum_{j=n+1}^{i_{1}+\cdots+i_{n+1}} \sum_{\sigma \in \mathcal{A}\left(i_{1}, \cdots, i_{n+1}, j\right)} P^{\sigma}\left(X_{0, s}^{i_{1}+\cdots+i_{n+1}-j} \otimes X_{s, t}^{j}\right),
$$

where $\mathcal{A}\left(i_{1}, \cdots, i_{n+1}, j\right)$ is a set of permutations of order $i_{1}+\cdots+i_{n+1}$ which fix the last component. We take the right hand side of (4.4) as the definition of $Y_{s, t}^{n+1 ; i_{1}, \cdots, i_{n+1}}$.

Therefore, for each $n \geqslant 1,1 \leqslant i_{1}, \cdots, i_{n} \leqslant N$ and $(s, t) \in \Delta$, we have defined $Y_{s, t}^{n ; i_{1}, \cdots, i_{n}} \in\left(\mathbb{R}^{d}\right)^{\otimes\left(i_{1}+\cdots+i_{n}\right)}$ from a purely algebraic point of view. In the case when one of those $i_{j}$ equals zero, we simply let $Y_{s, t}^{n ; i_{1}, \cdots, i_{n}}=0$. It remains to verify that this definition is exactly what we need. Such verification is a straight forward consequence of the fact that $\mathbf{X}$ is a geometric $p^{\prime}$-rough path for every $p^{\prime} \in(p,\lfloor p\rfloor+1)$ (c.f. [10], Corollary 8.24).

Proposition 4.1. Let $\mathbf{X} \in W G \Omega_{p}\left(\mathbb{R}^{d}\right)$ and $N \in \mathbb{N}$. Then the formula (4.3) together with

$$
Y_{s, t}^{n ; i_{1}, \cdots, i_{n}}=0 \text {, if one of } i_{j}=0,
$$

defines a multiplicative functional $\mathbb{Y}$ on the infinite dimensional tensor algebra $T\left(\left(E_{N}\right)\right)$ over $E_{N}$ which takes values in the group $G\left(E_{N}\right)$ of exponential Lie series over $E_{N}$. Moreover, for each $n \geqslant 1$,

$$
Y_{s, t}^{(n)}:=\left(1, Y_{s, t}^{1}, \cdots, Y_{s, t}^{n}\right),(s, t) \in \Delta,
$$

This article is protected by copyright. All rights reserved. 
has finite $p$-variation in the sense of Definition 2.1. Therefore, $Y^{(\lfloor p\rfloor)}$ defines a weakly geometric p-rough path $\mathbf{Y}$ over $E_{N}$ whose signature path is $\mathbb{Y}$ with first level path being the truncated signature path of $\mathbf{X}$ up to degree $N$.

Finally, the signature of $\mathbf{Y}$ is determined by the signature of $\mathbf{X}$ explicitly through the following formula:

$$
\begin{aligned}
& S(\mathbf{Y})_{0,1}^{n ; i_{1}, \cdots, i_{n}}
\end{aligned}
$$

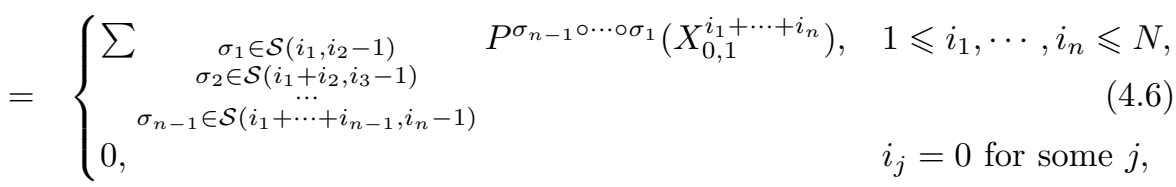

where each shuffle $\sigma_{j}$ is regarded as a permutation of order $i_{1}+\cdots+i_{n}$ by fixing the last $i_{j+1}+\cdots+i_{n}+1$ components.

Proof. Given $p^{\prime} \in(p,\lfloor p\rfloor+1)$, let $x_{n}$ be a sequence of continuous paths with bounded variation whose lifting to $G\lfloor p\rfloor\left(\mathbb{R}^{d}\right)$ converges to $\mathbf{X}$ in $p^{\prime}$-variation. The existence of $x_{n}$ is guaranteed by [10], Corollary 8.24. Since the truncated signature path $y_{n}$ of $x_{n}$ up to degree $N$ has bounded variation, it follows that each step in the previous formal calculation becomes exact equality when $\mathbf{X}$ is replaced by $x_{n}$. In other words, the signature path of $y_{n}$ given by iterated path integrals against $y_{n}$ coincides with the definition given in the previous formal construction. Therefore, it defines a multiplicative functional on $T\left(\left(E_{N}\right)\right)$ satisfying the shuffle product formula.

For each $n \geqslant 1$, since the free nilpotent group $G^{n}\left(E_{N}\right)$ of step $n$ over $E_{N}$ is a closed subset of $T^{(n)}\left(E_{N}\right)$, by the continuity of signature (c.f. [16], Theorem 2.2.2) we conclude the first part of the proposition. Moreover, from the explicit formula (4.3), it is easy to see that $Y_{s, t}^{n ; i_{1}, \cdots, i_{n}}$ has the following estimate:

$$
\left|Y_{s, t}^{n ; i_{1}, \cdots, i_{n}}\right| \leqslant C(N, n, \omega(0,1)) \cdot \omega(s, t)^{\frac{n}{p}},
$$

where

$$
\omega(s, t):=\sum_{i=1}^{n} \sup _{\mathcal{P}_{[s, t]}} \sum_{l}\left|X_{t_{l-1}, t_{l}}^{i}\right|^{\frac{p}{i}},(s, t) \in \Delta,
$$

and $C(N, n, \omega(0,1))$ is a constant depending only on $N, n, \omega(0,1)$. Therefore, $Y^{(n)}$ defined by (4.5) has finite $p$-variation and the conclusion of the second part holds. Finally, the formula (4.6) for the signature of $\mathbf{Y}$ can be seen directly by letting $s=0$ in the previous formal calculation (in this case the permutation sets $\mathcal{A}\left(i_{1}, \cdots, i_{n}, j\right)$ can be written down easily).

\subsection{Using Extended Signatures}

As mentioned before, we are going to reconstruct the tree-reduced path by using extended signatures. In particular, we will integrate along compactly supported one forms with continuous derivatives up to order $\alpha:=\lfloor p\rfloor+1$.

Let $\mathbf{X}$ be a weakly geometric $p$-rough path, and let $\left(\phi^{1}, \cdots, \phi^{n}\right)$ be a finite sequence of compactly supported $C^{\alpha}$-one forms on $\mathbb{R}^{d}$.

This article is protected by copyright. All rights reserved. 
Definition 4.1. The first level of the iterated path integral

$$
\int_{0<t_{1}<\cdots<t_{n}<1} \phi^{1}\left(d \mathbf{X}_{t_{1}}\right) \cdots \phi^{n}\left(d \mathbf{X}_{t_{n}}\right)
$$

is called the extended signature of $\mathbf{X}$ along $\left(\phi^{1}, \cdots, \phi^{n}\right)$, and it is denoted by $\left[\phi^{1}, \cdots, \phi^{n}\right](x)$.

In general, the extended signature of $\mathbf{X}$ along $\left(\phi^{1}, \cdots, \phi^{n}\right)$ can either be interpreted through the approximation

$$
\left[\phi^{1}, \cdots, \phi^{n}\right](x)=\lim _{k \rightarrow \infty} \int_{0<t_{1}<\cdots<t_{n}<1} \phi^{1}\left(d x_{t_{1}}^{(k)}\right) \cdots \phi^{n}\left(d x_{t_{n}}^{(k)}\right),
$$

where $x^{(k)}$ is a sequence of continuous paths with bounded variation whose lifting to $G\lfloor p\rfloor\left(\mathbb{R}^{d}\right)$ converges to $\mathbf{X}$ in $p^{\prime}$-variation for some $p^{\prime} \in(p,\lfloor p\rfloor+1)$, or through the unique solution to the rough differential equation

$$
\begin{cases}d x_{t}^{i}=d x_{t}^{i}, & 1 \leqslant i \leqslant d \\ d y_{t}^{j}=y_{t}^{j-1} \sum_{i=1}^{d} \phi_{i}^{j}\left(x_{t}\right) d x_{t}^{i}, & 1 \leqslant j \leqslant n \\ x_{0}=0, y_{0}=0 & \end{cases}
$$

where $y_{t}^{0}:=1$.

Remark 4.1. In the notation $\left[\phi^{1}, \cdots, \phi^{n}\right](x)$, we have used small " $x$ ", which denotes the first level path of $\mathbf{X}$, to emphasize that the integral is essentially constructed on $\mathbb{R}^{d}$ although the rigorous definition relies on the rough path nature of $\mathbf{X}$. Later on we will use extended signatures constructed on $E_{N}$ along one forms over $E_{N}$ for $N \geqslant\lfloor p\rfloor$.

Let $g$ be the signature of $\mathbf{X}$ and let $\phi$ be a compactly supported $C^{\alpha}$-one form. The starting point of our reconstruction is the crucial fact that the integral $\int_{0}^{1} \phi\left(d \mathbf{X}_{t}\right)$ can be explicitly reconstructed from the knowledge of $g$ and $\phi$.

Indeed, let $n_{0} \geqslant 1$ be such that $B_{n_{0}}=\left\{x \in \mathbb{R}^{d}:|x| \leqslant n_{0}\right\}$ contains the support of $\phi$. For each $n \geqslant n_{0}$, let $p_{n}$ be a polynomial one form such that

$$
\sup _{0 \leqslant j \leqslant \alpha} \sup _{B_{n}}\left|D^{j}\left(\phi-p_{n}\right)\right| \leqslant \frac{1}{n} .
$$

The existence of $p_{n}$ is guaranteed by the work of Bagby-Bos-Levenberg [2], Theorem 1. Moreover, from their proof the construction of $p_{n}$ is explicit.

Since $p_{n}$ is a polynomial one form, the integral $\int_{0}^{1} p_{n}\left(d \mathbf{X}_{t}\right)$ can be directly computed from $g$ using the shuffle product formula. Now it remains to show the following simple fact.

Proposition 4.2. The value of the integral $\int_{0}^{1} \phi\left(d \mathbf{X}_{t}\right)$ is given by

$$
\int_{0}^{1} \phi\left(d \mathbf{X}_{t}\right)=\lim _{n \rightarrow \infty} \int_{0}^{1} p_{n}\left(d \mathbf{X}_{t}\right) .
$$

This article is protected by copyright. All rights reserved. 
Proof. Since $x([0,1])$ is a compact subset of $\mathbb{R}^{d}$, we know that $x([0,1]) \subset \subset B_{n_{1}}$ for some $n_{1} \geqslant n_{0}$. Therefore,

$$
\sup _{0 \leqslant j \leqslant \alpha B_{n_{1}}} \sup _{0}\left|D^{j}\left(\phi-p_{n}\right)\right|<\frac{1}{n}, \forall n \geqslant n_{1} .
$$

The result follows from the continuity of the integration map $\phi \mapsto \int_{0}^{1} \phi\left(d \mathbf{X}_{t}\right)$ (c.f. [10], Theorem 10.47). Note that here the path $\mathbf{X}$ is fixed, thus the continuity holds under the $C^{\alpha}$-norm over $B_{n_{1}}$.

The same argument applies to extended signatures. In particular, for a given finite sequence $\left(\phi^{1}, \cdots, \phi^{n}\right)$ of compactly supported $C^{\alpha}$-one forms, the extended signature $\left[\phi^{1}, \cdots, \phi^{n}\right](x)$ can be reconstructed from the knowledge of $g$ and these one forms.

In our reconstruction problem, as we have pointed out before, we shall integrate over $E_{\lfloor p\rfloor}$ rather than over $\mathbb{R}^{d}$. More generally, given $N \geqslant\lfloor p\rfloor$, let $y$ be the truncated signature path of $\mathbf{X}$ up to some degree $N$, and let $\mathbf{Y}$ be the weakly geometric $p$-rough path over $E_{N}$ defined in Proposition 4.1. We know that the signature of $\mathbf{Y}$ is determined by $g$ through the formula (4.6). Therefore, given a finite sequence $\left(\Phi^{1}, \cdots, \Phi^{n}\right)$ of compactly supported $C^{\alpha}$-one forms on $E_{N}$, the extended signature $\left[\Phi^{1}, \cdots, \Phi^{n}\right](y)$ can be reconstructed from the knowledge of $g$ and these one forms on $E_{N}$.

\section{The Reconstruction: Non-self-intersecting Case}

In this section, we develop our reconstruction for the case when the tree-reduced weakly geometric $p$-rough paths are simple. Although we could treat the general case in one go, a good understanding of the non-self-intersecting case is very helpful.

Recall that

$$
E_{\lfloor p\rfloor}=\bigoplus_{i=0}^{\lfloor p\rfloor}\left(\mathbb{R}^{d}\right)^{\otimes i}
$$

is an Euclidean space of dimension $D=1+d+\cdots+d^{\lfloor p\rfloor}$ with Euclidean norm given by

$$
\|\cdot\|_{E_{\lfloor p\rfloor}}=\sum_{i=0}^{\lfloor p\rfloor}\|\cdot\|_{\left(\mathbb{R}^{d}\right)^{\otimes i}} .
$$

Elements in $E_{\lfloor p\rfloor}$ are of the form $a=\left(a^{I}\right)_{0 \leqslant|I| \leqslant\lfloor p\rfloor}$, where $I$ is a word over the alphabet $\{1, \cdots, d\}$. Here the order of coordinates $a^{I}$ in a homogeneous tensor product $\left(\mathbb{R}^{d}\right)^{\otimes k}$ is not important, but the increasing order with respect to the tensor degree is important (in order to make use of the decay of signature as we will see in Section 6).

In the rest of this section, we assume that $\mathbf{X}$ is a simple tree-reduced weakly geometric $p$-rough path with signature $g$.

This article is protected by copyright. All rights reserved. 


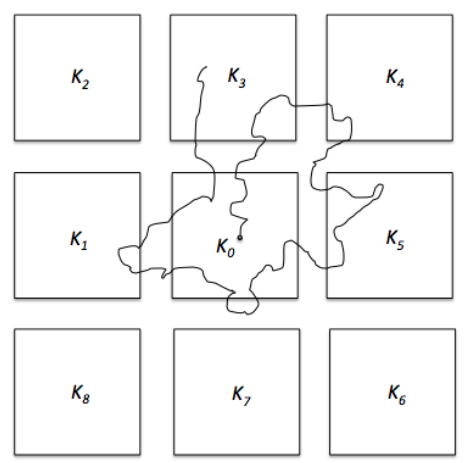

Figure 5.1: This figure illustrates the construction of the discrete route of a path in dimension 2. In this example $L=7$, and the discrete route of the underlying path is given by the word $(0,3,4,5,0,1,0,3)$.

\subsection{Recovering the Discrete Route in a Given Geometric Scheme}

Let $\left\{K_{0}, \cdots, K_{r}\right\}$ be a finite family of bounded domains in $E_{\lfloor p\rfloor}$ whose closures are mutually disjoint. Suppose that the path $\mathbf{X}$ starts in $K_{0}$.

We define $L$ to be the total number of domains $K_{i}$ visited by $\mathbf{X}$ in order (excluding the initial one $K_{0}$ ). $L$ is finite from the continuity of $\mathbf{X}$ and the disjointness of those $\overline{K_{i}}$. Let

$$
\left\{\left(\tau_{k}, m_{k}\right) \in[0,1] \times\{0, \cdots, r\}: 0 \leqslant k \leqslant L\right\}
$$

be the corresponding sequence of entry times together with domains visited, where $\tau_{0}=0$ and $m_{0}=0$. Here we label the domains by their subscripts $\{0, \cdots, r\}$.

We should point out that revisiting the same domain before entering other ones does not count, but revisiting after entering some other domain does count. Moreover, we only consider entrance of these domains (which are open) but do not consider the case of bouncing at the boundaries.

Figure 5.1 illustrates the notions in dimension 2. In this example $L=7$, and the discrete route of the underlying path is given by the word $(0,3,4,5,0,1,0,3)$.

The next step is to show that in the non-self-intersecting case, the ordered sequence $\left(m_{0}, \cdots, m_{L}\right)$ of domains visited by $\mathbf{X}$ can be reconstructed from the signature $g$ through computing extended signatures.

To be more precise, we have the following result. The construction of the one forms involved is motivated from the work of [4].

Proposition 5.1. Let $S=\left\{m_{0}, \cdots, m_{L}\right\} \subset\{0, \cdots, r\}$ be the associated set of domains visited by $\mathbf{X}$.

This article is protected by copyright. All rights reserved. 
(1) For each $m \in S$, there exists a $C^{\alpha}$-one form $\Phi_{m}$ supported on $\overline{K_{m}}$, such that the extended signature

$$
\left[\Phi_{m_{0}}, \cdots, \Phi_{m_{L}}\right](\mathbf{X}) \neq 0 .
$$

Here we should regard $\mathbf{X}$ as the first level of the rough path $\mathbf{Y}$ in Proposition 4.1 with $N=\lfloor p\rfloor$, and the extended signature is constructed on $E_{\lfloor p\rfloor}$ for $\mathbf{Y}$ (see the last paragraph of Section 4).

(2) For $0 \leqslant m \leqslant r$, let $\Psi_{m}$ be any given $C^{\alpha}$-one form supported on $\overline{K_{m}}$. Suppose that $\mathbf{n}=\left(n_{0}=0, n_{1}, \cdots, n_{l}\right)$ is an arbitrary word over the alphabet $\{0, \cdots, r\}$ such that $n_{k} \neq n_{k-1}$ for $1 \leqslant k \leqslant l$. If $n_{k} \notin S$ for some $1 \leqslant k \leqslant l$, or if it is different from the word $\mathbf{m}=\left(m_{0}, \cdots, m_{L}\right)$ when $l \geqslant L$, then the extended signature

$$
\left[\Psi_{n_{0}}, \cdots, \Psi_{n_{l}}\right](\mathbf{X})=0 .
$$

Proof. Given $m \in S$, let $0 \leqslant k \leqslant L$ be such that $m_{k}=m$. By the definition of $\tau_{k}$, there exist $\tau_{k}<s<t<\tau_{k+1}$ such that $\operatorname{Im}\left(\left.\mathbf{X}\right|_{[s, t]}\right) \subset K_{m}$ (here we set $\left.\tau_{L+1}=1\right)$. Let $s^{\prime}, t^{\prime}$ satisfy $s<s^{\prime}<t^{\prime}<t$. Since $\mathbf{X}$ is simple, we know that

$$
\operatorname{Im}\left(\left.\mathbf{X}\right|_{\left[s, s^{\prime}\right]}\right) \bigcap \operatorname{Im}\left(\left.\mathbf{X}\right|_{\left[t^{\prime}, t\right]}\right)=\emptyset
$$

and

$$
\operatorname{Im}\left(\left.\mathbf{X}\right|_{\left[s^{\prime}, t^{\prime}\right]}\right) \bigcap \operatorname{Im}\left(\left.\mathbf{X}\right|_{\left[\tau_{k}, s\right] \cup\left[t, \tau_{k+1}\right]}\right)=\emptyset .
$$

It follows that there exist open neighborhoods $U_{1}, U_{2}, V_{1}, V_{2}$ of $\operatorname{Im}\left(\left.\mathbf{X}\right|_{\left[s, s^{\prime}\right]}\right)$, $\operatorname{Im}\left(\left.\mathbf{X}\right|_{\left[t^{\prime}, t\right]}\right), \operatorname{Im}\left(\left.\mathbf{X}\right|_{\left[s^{\prime}, t^{\prime}\right]}\right), \operatorname{Im}\left(\left.\mathbf{X}\right|_{\left[\tau_{k}, s\right] \cup\left[t, \tau_{k+1}\right]}\right)$ respectively, such that

$$
U_{1} \bigcup V_{1} \bigcup U_{2} \subset \subset K_{m}
$$

and

$$
U_{1} \bigcap U_{2}=V_{1} \bigcap V_{2}=\emptyset
$$

Let $F, G$ be two $C^{\alpha}$-functions on $E_{\lfloor p\rfloor}$ such that

$$
F=0 \text { on } U_{1}, F=1 \text { on } U_{2},
$$

and

$$
G=0 \text { on } V_{2}, G=1 \text { on } V_{1},
$$

respectively. We define $\Phi_{k}=G d F$. From the construction it is straight forward to see that

$$
\int_{\tau_{k}}^{\tau_{k+1}} \Phi_{k}\left(d \mathbf{Y}_{t}\right)=F\left(\mathbf{X}_{t^{\prime}}\right)-F\left(\mathbf{X}_{s^{\prime}}\right)=1 .
$$

Moreover, the value of the integral depends only on the definition of $\Phi_{k}$ on $U_{1} \cup U_{2} \cup V_{1} \cup V_{2}$. Together with the fact that $\Phi_{k}=0$ on $V_{2}$ and (5.1), we can certainly modify the definition of $\Phi_{k}$ without changing its values on $U_{1} \cup U_{2} \cup$ $V_{1} \cup V_{2}$ so that it is supported on $\overline{K_{m}}$. Furthermore, it follows again from the simpleness of $\mathbf{X}$ that the family

$$
\left\{\operatorname{Im}\left(\left.\mathbf{X}\right|_{\left[\tau_{k}, \tau_{k+1}\right]}\right): 0 \leqslant k \leqslant L \text { with } m_{k}=m\right\}
$$


are mutually disjoint. Therefore, by choosing $U_{1} \cup U_{2} \cup V_{1} \cup V_{2}$ small enough, we can define a single $C^{\alpha}$-one form $\Phi_{m}$ supported on $\overline{K_{m}}$, such that

$$
\int_{\tau_{k}}^{\tau_{k+1}} \Phi_{m}\left(d \mathbf{Y}_{t}\right)=1
$$

for every $0 \leqslant k \leqslant L$ satisfying $m_{k}=m$. The first part of the lemma follows from the decomposition of the extended signature:

$$
\left[\Phi_{m_{0}}, \cdots, \Phi_{m_{L}}\right](\mathbf{X})=\prod_{k=0}^{L} \int_{\tau_{k}}^{\tau_{k+1}} \Phi_{m_{k}}\left(d \mathbf{Y}_{t}\right)
$$

given in [3], Lemma 5.1 (1).

The second part follows from [3], Lemma 4.2 and Lemma 5.1 (2), (3).

Remark 5.1. The second part of Proposition 5.1 does not depend on the nonself-intersecting assumption; it is true for all weakly geometric $p$-rough paths.

The construction of the one forms in Proposition 5.1 certainly depends on the trajectory of $\mathbf{X}$ in a crucial way. However, once existence is guaranteed by the result of Proposition 5.1, we actually do not need the specific construction and it is sufficient to compute extended signatures with respect to a pre-specified countable generating set for the space of one forms. This is a consequence of separability and continuity.

Firstly, as $\alpha=\lfloor p\rfloor+1$ is a positive integer, it is well known that the space of $C^{\alpha}$-functions supported on some given compact set $K$ equipped with the $C_{K}^{\alpha}$-topology is separable. Therefore, for each domain $K_{i}$, we can specify a countable dense subset $\left\{\Phi_{n}^{(i)}: n \geqslant 1\right\}$ of the space of $C^{\alpha}$-one forms supported on $\overline{K_{i}}$. If the geometry of $K_{i}$ is simple, the construction of $\Phi_{n}^{(i)}$ can be made explicit for instance by using wavelets. In fact in our situation the domains $K_{i}$ are just cubes or convex hulls of two concentric cubes.

Secondly, for given $l \geqslant 0$, let $\mathcal{W}_{l}$ be the set of words $\mathbf{n}=\left(n_{0}=0, n_{1}, \cdots, n_{l}\right)$ such that $n_{k} \neq n_{k-1}$ for $1 \leqslant k \leqslant l$. Given a word $\mathbf{n} \in \mathcal{W}_{l}$, let $\left\{I_{m}(g ; l, \mathbf{n}): m \geqslant\right.$ $1\}$ be an enumeration of all possible extended signatures of $\mathbf{Y}$ along the word $\mathbf{n}$ with respect to the previous specification of generating one forms. Define

$$
\chi(g ; l, \mathbf{n})= \begin{cases}1, & \text { if } I_{m}(g ; l, \mathbf{n}) \neq 0 \text { for some } m \\ 0, & \text { otherwise }\end{cases}
$$

Note that $\chi(g ; l, \mathbf{n})$ is determined by the signature $g$ and the word $\mathbf{n} \in \mathcal{W}_{l}$. According to the continuity of rough path integrals along one forms, a direct consequence of Proposition 5.1 is the following.

Corollary 5.1. The total number $L$ of domains visited by $\mathbf{X}$ is given by

$$
L=\sup \left\{l \geqslant 0: \chi(g ; l, \mathbf{n})=1 \text { for some } \mathbf{n} \in \mathcal{W}_{l}\right\},
$$

Moreover, there is one and only one word $\mathbf{n}=\left(n_{0}, \cdots, n_{L}\right) \in \mathcal{W}_{L}$ such that $\chi(g ; l, \mathbf{n})=1$, and $\mathbf{n}$ is exactly the word $\mathbf{m}=\left(m_{0}, \cdots, m_{L}\right)$ corresponding to the ordered sequence of domains visited by $\mathbf{X}$.

This article is protected by copyright. All rights reserved. 
In other words, in the case when $\mathbf{X}$ is simple, the word $\mathbf{m}$ can be reconstructed from the signature of $\mathbf{X}$.

Remark 5.2. In contrast to the probabilistic setting, here as we have to treat every path equally, a universal construction of a single one form for all paths is not possible. Instead we need to compute extended signatures along all possible "directions" to reveal geometric information about the underlying path. This is the nature of the problem if we aim at finding a universal reconstruction for the class of all tree-reduced weakly geometric $p$-rough paths in one go.

\subsection{The Key Ingredient: A Stable Quantity on Words}

Now we turn to the reconstruction of $\mathbf{X}$ by constructing our geometric scheme in a more specific way.

Recall that $E_{\lfloor p\rfloor}$ is an Euclidean space of dimension $D$. For $0 \leqslant j \leqslant D$, let $V_{j}$ be the set of points $z=\left(z^{I}\right)_{0 \leqslant|I| \leqslant\lfloor p\rfloor} \in E_{\lfloor p\rfloor}$ such that exactly $j$ of those $z^{I}$ are integers and the rest are half-integers (i.e. of the form $z^{I}=n / 2$ where $n$ is an odd integer).

Definition 5.1. For $0 \leqslant j \leqslant D$, the open $j$-skeleton $K_{j}$ is defined to be the disjoint union

$$
K_{j}=\bigcup_{z \in V_{j}}\left\{a \in E_{\lfloor p\rfloor}:\left|a^{I}-z^{I}\right|<\frac{1}{2} \text { if } z^{I} \text { is an integer and } a^{I}=z^{I} \text { otherwise }\right\}
$$

of $j$-dimensional open faces with centers in $V_{j}$, and the closed $j$-skeleton $C_{j}$ is defined to be

$$
C_{j}=\bigcup_{i=0}^{j} K_{i} .
$$

Figure 5.2 illustrates the definition of the open and closed skeletons when $D=2$. Here $K_{2}$ consists of the set of open cubes centered at integer points, $K_{1}$ consists of their open edges and $K_{0}$ is the set of vertices.

From now on, we fix $\varepsilon>0$ for the rest of this subsection, and for simplicity in the upcoming notions the dependence on $\varepsilon$ will be omitted. Correspondingly, the skeletons $K_{j}$ and $C_{j}$ are scaled to the $\varepsilon$ order.

Let $\delta$ be a parameter with $\delta<\varepsilon$. For convenience we should think of $\varepsilon, \delta$ as discrete parameters (for instance we can simply take $\varepsilon=1 / m, \delta=1 / n$ with $n>m)$. The same remark applies to other parameters to be introduced later on.

For $z \in V_{D}$, let $H_{z}^{\delta ; D}$ be the cube defined by

$$
H_{z}^{\delta ; D}=\left\{a \in E_{\lfloor p\rfloor}:\left|a^{I}-\varepsilon z^{I}\right|<\frac{\varepsilon-\delta}{2}\right\},
$$

The geometric scheme of the collection $\left\{\mathbf{1}+H_{z}^{\delta ; D}: z \in V_{D}\right\}(\mathbf{1}=(1,0, \cdots, 0)$ is the unit of $\left.E_{\lfloor p\rfloor}\right)$ is denoted by $\mathcal{C}^{\delta ; D}$. In other words, $\mathcal{C}^{\delta ; D}$ consists of cubes with edge length $\varepsilon-\delta$ and narrow tunnels with width $\delta$. 


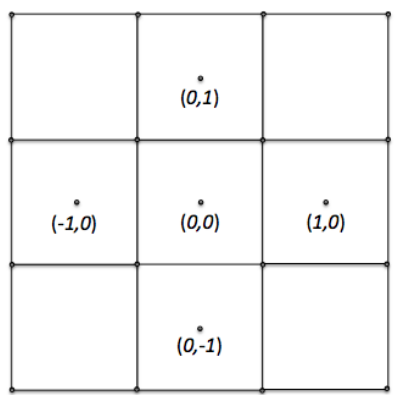

Figure 5.2: This figure illustrates the definition of the open and closed skeletons in when $D=2$. Here $K_{2}$ consists of the set of open cubes centered at integer points, $K_{1}$ consists of their open edges and $K_{0}$ is the set of vertices.

We define $L^{\delta ; D}$ and $\left\{\tau_{k}^{\delta ; D}, m_{k}^{\delta ; D}: 0 \leqslant k \leqslant L^{\delta ; D}\right\}$ for the geometric scheme $\mathcal{C}^{\delta ; D}$ in the same way as in the last subsection. Here we label the domains in $\mathcal{C}^{\delta ; D}$ by elements in $V_{D}$ so that $m_{k}^{\delta ; D} \in V_{D}$ is the center of the $k$-th visited cube. According to the previous discussion, the word

$$
\mathbf{m}^{\delta ; D}=\left(m_{0}^{\delta ; D}, \cdots, m_{L^{\delta ; D}}^{\delta ; D}\right)
$$

can be reconstructed from the signature $g$. Associated with the word $\mathbf{m}^{\delta ; D}$, we can construct a polygonal path by connecting the centers $m_{k}^{\delta ; D}\left(0 \leqslant k \leqslant L^{\delta ; D}\right)$ in order.

Remark 5.3. Although $\mathcal{C}^{\delta ; D}$ contains countably many cubes, the discussion in the last subsection certainly carries through in without any difficulty since from compactness we know that the underlying path can visit at most finitely many different cubes.

As we have pointed out in the discussion in Section 3, we cannot expect the convergence of the polygonal approximation to our underlying path in any sense when we let $\varepsilon, \delta \rightarrow 0$. The main idea of overcoming this issue is to let the signature $g$ choose the "right" width $\delta(g)$, so that in the resulting geometric scheme $\mathcal{C}^{\delta(g) ; D}$, we can conclude that if the underlying path $\mathbf{X}$ has a long excursion in the complement of $\mathcal{C}^{\delta(g) ; D}$ (i.e. the tunnels) during some time period $[s, t]$, it has to spend some time period $\left[s^{\prime}, t^{\prime}\right] \subset[s, t]$ having such a long excursion in the closed $(D-1)$-skeleton. This enables us to start a recursive construction until we reach the 0 -skeleton by adding more and more domains over the open skeleton in each dimension. The conclusion is that in the final geometric scheme $\mathcal{C}$, the path is not able to have a long excursion in the complement of $\mathcal{C}$ and the convergence is then immediate.

The way of choosing $\delta$ by the signature is through a stable quantity on words.

Let $\mathcal{W}^{D}$ be the set of all finite words $\mathbf{z}=\left(z_{0}=0, z_{1}, \cdots, z_{n}\right)$ over the alphabet $V_{D}$ such that $z_{k} \neq z_{k-1}$ for all $k$.

This article is protected by copyright. All rights reserved. 
Definition 5.2. A stable quantity over $V_{D}$ with respect to the geometric schemes $\mathcal{C}^{\delta ; D}(0<\delta<\varepsilon)$ is a map $\mathfrak{s}: \mathcal{W}^{D} \rightarrow \mathbb{Z}$, such that for any continuous path $x$ in $E_{\lfloor p\rfloor}$ starting at $\mathbf{1}$, the limit

$$
\lim _{\delta \rightarrow 0} \mathfrak{s}\left(\mathbf{m}^{\delta ; D}\right)
$$

always exists, where $\mathbf{m}^{\delta ; D}$ is the word corresponding to the ordered sequence of cubes visited by $x$ in the geometric scheme $\mathcal{C}^{\delta ; D}$ defined as before.

Example 5.1. Given a word $\mathbf{z}=\left(z_{0}, \cdots, z_{n}\right) \in \mathcal{W}^{D}$, let $S_{\mathbf{z}}=\left\{z_{0}, \cdots, z_{n}\right\} \subset$ $V_{D}$ be the associated set of letters in $\mathbf{z}$. Define $\mathfrak{s}(\mathbf{z})=\sharp\left(S_{\mathbf{z}}\right)$ (the total number of elements in $S_{\mathbf{z}}$ ). Then $\mathfrak{s}$ defines a stable quantity. Indeed, let $x$ be a continuous path $x$ starting at $\mathbf{1}$. For $\delta_{1}<\delta_{2}$, it is easy to see that $\mathbf{m}^{\delta_{2} ; D}$ is a subword of $\mathbf{m}^{\delta_{1} ; D}$, and thus $\mathfrak{s}\left(\mathbf{m}^{\delta_{2} ; D}\right) \leqslant \mathfrak{s}\left(\mathbf{m}^{\delta_{1} ; D}\right)$. Moreover, from the boundedness of $x$ we know that

$$
\sup _{0<\delta<\varepsilon} \mathfrak{s}\left(\mathbf{m}^{\delta ; D}\right)<\infty .
$$

Therefore the limit (5.3) exists. However, this stable quantity is not applicable for our purpose.

Example 5.2. For $\mathbf{z}=\left(z_{0}, \cdots, z_{n}\right) \in \mathcal{W}^{D}$, define $\mathfrak{s}(\mathbf{z})=n$ (the length of $\mathbf{z}$ ). Then $\mathfrak{s}$ is not a stable quantity. This is simply because we can construct a path $x$, such that the length of the associated word $\mathbf{m}^{\delta ; D}$ explodes as $\delta \rightarrow 0$ (think of the topologist's sine curve in higher dimensions and note that the distance between neighboring cubes tends to zero).

Now we are going to define a stable quantity $\mathfrak{s}_{D}$, such that the width parameter

$$
\delta_{1}:=\frac{1}{2} \sup \left\{0<\delta<\varepsilon: \mathfrak{s}_{D}\left(\mathbf{m}^{\delta^{\prime} ; D}\right)=\text { const. } \forall \delta^{\prime} \leqslant \delta\right\}>0
$$

obtained from the stability of $\mathfrak{s}_{D}\left(\mathrm{~m}^{\delta ; D}\right)$ as $\delta \rightarrow 0$ will serve our purpose. In particular we know that $\mathfrak{s}_{D}\left(\mathbf{m}^{\delta^{\prime} ; D}\right)=\mathfrak{s}_{D}\left(\mathbf{m}^{\delta_{1} ; D}\right)$ for all $\delta^{\prime} \leqslant \delta_{1}$. Note that $\delta_{1}$ is determined by the signature $g$ explicitly.

Definition 5.3. Let $\mathbf{z}=\left(z_{0}, \cdots, z_{n}\right) \in \mathcal{W}^{D}$. An admissible chain $c$ in $\mathbf{z}$ is a subword $\left(z_{i_{1}}, \cdots, z_{i_{r}}\right)$ for some $0 \leqslant i_{1}<\cdots<i_{r} \leqslant n$ such that

$$
\left|z_{i_{k}}-z_{i_{k-1}}\right| \geqslant 2 \sqrt{D}
$$

for all $2 \leqslant k \leqslant r$.

For $\mathbf{z} \in \mathcal{W}^{D}$, we define $\mathfrak{s}_{D}(\mathbf{z})$ to be the maximal length $r$ of all possible admissible chains in $\mathbf{z}$ (if admissible chains do not exist, we simply define $\left.\mathfrak{s}_{D}(\mathbf{z})=1\right)$. It is obvious that $\mathfrak{s}_{D}(\mathbf{z})$ is well-defined. Any admissible chain in $\mathbf{z}$ with length $\mathfrak{s}_{D}(\mathbf{z})$ is called a maximal admissible chain. Note that maximal admissible chains may not be unique.

This article is protected by copyright. All rights reserved. 
Example 5.3. Consider the case when $D=2$. Let

$$
\mathbf{z}=((0,0),(0,1),(1,1),(2,1),(2,2),(3,1)) .
$$

Then $\mathfrak{s}_{D}(\mathbf{z})=2$, and $((0,0),(2,2)),((0,0),(3,1)),((0,1),(3,1))$ are all maximal admissible chains.

Now we have the following result.

Lemma 5.1. $\mathfrak{s}_{D}$ is a stable quantity.

Proof. Let $x$ be a continuous path starting at 1 . For $0<\delta<\varepsilon$, let $c^{\delta}=$ $\left(z_{i_{1}}, \cdots, z_{i_{r}}\right)$ be an admissible chain of $\mathbf{m}^{\delta ; D}$ such that $r=\mathfrak{s}_{D}\left(\mathbf{m}^{\delta ; D}\right)$. It follows that $c^{\delta}$ is also an admissible chain of $\mathbf{m}^{\delta^{\prime} ; D}$ for any $\delta^{\prime}<\delta$ since $\mathbf{m}^{\delta ; D}$ is a subword of $\mathbf{m}^{\delta^{\prime} ; D}$. Therefore, $\mathfrak{s}_{D}\left(\mathbf{m}^{\delta^{\prime} ; D}\right) \geqslant \mathfrak{s}_{D}\left(\mathbf{m}^{\delta ; D}\right)$. On the other hand, since $x$ is continuous, there exists $\eta>0$ such that

$$
\left|x_{t}-x_{s}\right| \geqslant \sqrt{D} \varepsilon \Longrightarrow|t-s| \geqslant \eta
$$

In the geometric scheme $\mathcal{C}^{\delta ; D}$, let $\zeta_{1}<\cdots<\zeta_{r}$ be a sequence of entry times corresponding to the admissible chain $c^{\delta}$ (they exist by definition). Then for each $k$ we have

$$
\begin{aligned}
\left|x_{\zeta_{k}}-x_{\zeta_{k-1}}\right| & \geqslant\left|\varepsilon z_{i_{k}}-\varepsilon z_{i_{k-1}}\right|-\left|x_{\zeta_{k}}-\left(\mathbf{1}+\varepsilon z_{i_{k}}\right)\right|-\left|x_{\zeta_{k-1}}-\left(\mathbf{1}+\varepsilon z_{i_{k-1}}\right)\right| \\
& \geqslant 2 \sqrt{D} \varepsilon-\frac{\sqrt{D}}{2} \varepsilon-\frac{\sqrt{D}}{2} \varepsilon \\
& =\sqrt{D} \varepsilon .
\end{aligned}
$$

It follows that $\left|\zeta_{k}-\zeta_{k-1}\right| \geqslant \eta$ for every $k$, and thus $r \leqslant 1 / \eta+1$. Therefore, the limit (5.3) exists.

Now we define $\delta_{1}$ by the formula (5.4).

The next step is to develop similar construction for geometric schemes over the open $(D-1)$-skeleton.

Suppose $0<\delta \leqslant \delta_{1}$. For $z \in V_{D-1}$, define

$$
\begin{aligned}
H_{z}^{\delta ; D-1}= & \left\{a \in E_{\lfloor p\rfloor}:\left|a^{I}-\varepsilon z^{I}\right|<\frac{\varepsilon-\delta}{2} \text { if } z^{I}\right. \text { is an integer } \\
& \text { and } \left.\left|a^{I}-\varepsilon z^{I}\right|<\frac{\delta}{4} \text { otherwise }\right\} .
\end{aligned}
$$

Let $C_{z}^{\delta ; D-1}$ be the convex hull of the two cubes $H_{z}^{\delta ; D-1}$ and $H_{z}^{\delta_{1} ; D-1}$. We denote the geometric scheme of $\left\{\mathbf{1}+C_{z}^{\delta ; D-1}: z \in V_{D-1}\right\}$ by $\mathcal{C}^{\delta ; D-1}$. Figure 5.3 (excluding the 4 cubes) illustrates the construction of $\mathcal{C}^{\delta ; D-1}$ when $D=2$.

Lemma 5.2. (1) For each $\delta \leqslant \delta_{1},\left\{\overline{C_{z}^{\delta ; D-1}}: z \in V_{D-1}\right\}$ are mutually disjoint.

(2) For each $z \in V_{D-1}$, if $\delta^{\prime}<\delta \leqslant \delta_{1}$, then $\overline{C_{z}^{\delta ; D-1}} \subset \overline{C_{z}^{\delta^{\prime} ; D-1}}$.

This article is protected by copyright. All rights reserved. 


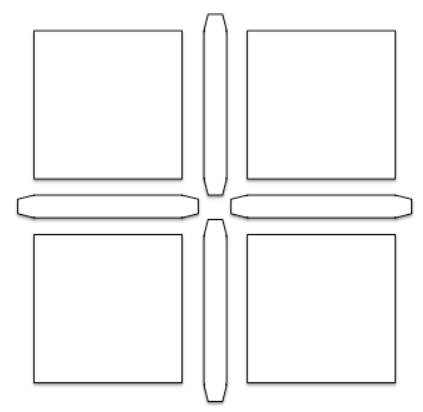

Figure 5.3: This figure illustrates the construction of $\mathcal{C}^{\delta ; D-1}$ and $\mathcal{C}^{\varepsilon}(g)$ (to be defined later) when $D=2$.

Proof. (1) Assume that $a \in \overline{C_{z}^{\delta ; D-1}} \cap \overline{C_{z^{\prime}}^{\delta ; D-1}}$ for some $z \neq z^{\prime} \in V_{D-1}$. Let $\mathcal{I}, \mathcal{I}^{\prime}$ be the sets of components of $z, z^{\prime}$ which are integers respectively. If $\mathcal{I}=\mathcal{I}^{\prime}$, then for any $I$ we have

$$
\left|a^{I}-\varepsilon z^{I}\right| \leqslant \frac{\varepsilon-\delta}{2},\left|a^{I}-\varepsilon\left(z^{\prime}\right)^{I}\right| \leqslant \frac{\varepsilon-\delta}{2}
$$

if $I \in \mathcal{I}$, which implies that $z^{I}=\left(z^{\prime}\right)^{I}$, and

$$
\left|a^{I}-\varepsilon z^{I}\right| \leqslant \frac{\delta}{4},\left|a^{I}-\varepsilon\left(z^{\prime}\right)^{I}\right| \leqslant \frac{\delta}{4}
$$

if $I \notin \mathcal{I}$, which also implies that $z^{I}=\left(z^{\prime}\right)^{I}$. This contradicts $z \neq z^{\prime}$. If $\mathcal{I} \neq \mathcal{I}^{\prime}$, there exists some $I$ such that $I \in \mathcal{I} \cap\left(\mathcal{I}^{\prime}\right)^{c}$. It follows that $\left|z^{I}-\left(z^{\prime}\right)^{I}\right| \geqslant 1 / 2$. On the other hand, we have

$$
\left|a^{I}-\varepsilon z^{I}\right| \leqslant \frac{\varepsilon-\delta}{2},\left|a^{I}-\varepsilon\left(z^{\prime}\right)^{I}\right| \leqslant \frac{\delta}{4} .
$$

Therefore,

$$
\left|z^{I}-\left(z^{\prime}\right)^{I}\right| \leqslant \frac{1}{2}-\frac{\delta}{4 \varepsilon}
$$

which is contradiction

(2) First note that $\overline{H_{z}^{\delta ; D-1}}$ is the convex hull of its vertices. Therefore it suffices to show that each vertex of $\overline{H_{z}^{\delta ; D-1}}$ is an element of $\overline{C_{z}^{\delta^{\prime} ; D-1}}$. Let $a$ be a vertex of $\overline{H_{z}^{\delta ; D-1}}$. Without loss of generality, we may assume that

$$
a^{I}= \begin{cases}\varepsilon z^{I}+\frac{\varepsilon-\delta}{2}, & \text { if } I \in \mathcal{I} ; \\ \varepsilon z^{I}+\frac{\delta}{4}, & \text { otherwise, }\end{cases}
$$

where $\mathcal{I}$ is the set of components of $z$ which are integers. Let $a^{(0)}$ and $a^{(1)}$ be the corresponding vertices of $\overline{H_{z}^{\delta_{1} ; D-1}}$ and $\overline{H_{z}^{\delta^{\prime} ; D-1}}$ respectively (i.e. replacing 
$\delta$ by $\delta_{1}$ and $\delta^{\prime}$ in (5.6) respectively). From direct calculation we know that

$$
a=\frac{\delta-\delta^{\prime}}{\delta_{1}-\delta^{\prime}} a^{(0)}+\frac{\delta_{1}-\delta}{\delta_{1}-\delta^{\prime}} a^{(1)} .
$$

Therefore $a \in \overline{C_{z}^{\delta^{\prime} ; D-1}}$.

Lemma 5.2 enables us to develop the same construction over the geometric scheme $\mathcal{C}^{\delta ; D-1}$ as we have done over $\mathcal{C}^{\delta ; D}$. More precisely, we define $L^{\delta ; D-1}$, $\tau_{k}^{\delta ; D-1}, \mathbf{m}^{\delta ; D-1}$, admissible chains, and the quantity $\mathfrak{s}_{D-1}$ for words in $\mathcal{W}^{D-1}$ over the alphabet $V_{D-1}$ in the same way as before (here we allow the empty word as the path may not necessarily visit $\mathcal{C}^{\delta ; D-1}$, and we set $\mathfrak{s}_{D-1}(\emptyset)=0$ in this case). Moreover, the same argument as in the proof of Lemma 5.1 shows that $\mathfrak{s}_{D-1}$ is a stable quantity over $V_{D-1}$ with respect to the geometric schemes $\mathcal{C}^{\delta ; D-1}\left(0<\delta \leqslant \delta_{1}\right)$. Finally we define

$$
\delta_{2}=\frac{1}{2} \sup \left\{0<\delta<\delta_{1}: \mathfrak{s}_{D-1}\left(\mathbf{m}^{\delta^{\prime} ; D-1}\right)=\text { const. } \forall \delta^{\prime} \leqslant \delta\right\}>0
$$

as in (5.4).

Remark 5.4. We construct the convex hull of $H_{z}^{\delta ; D-1}$ and $H_{z}^{\delta_{1} ; D-1}$ instead of simply using the cube $H_{z}^{\delta ; D-1}$ because $\mathfrak{s}_{D-1}$ will no longer be a stable quantity if we use the latter. Moreover, it will be difficult to show stability for other attempts to construct a stable quantity without property (2) of Lemma 5.2.

We carry on the construction recursively. Assume that we have constructed $\mathcal{C}^{\delta_{j} ; D-j+1}$ for $j=1, \cdots, i$. For $\delta \leqslant \delta_{i}$ and $z \in V_{i}$, we construct $H_{z}^{\delta ; D-i}$ by (5.5) and define $C_{z}^{\delta ; D-i}$ to be the convex hull of $H_{z}^{\delta ; D-i}$ and $H_{z}^{\delta_{i} ; D-i}$. The geometric scheme of $\left\{\mathbf{1}+C_{z}^{\delta ; D-i}: z \in V_{D-i}\right\}$ is denoted by $\mathcal{C}^{\delta ; D-i}$. Lemma 5.2 holds in exactly the same way as before. Moreover, we construct the stable quantity $\mathfrak{s}_{D-i}$ over $V_{D-i}$ and define $\delta_{i+1}>0$ accordingly from the stability property.

Therefore, we obtain $D$ geometric schemes $\mathcal{C}^{\delta_{i} ; D-i+1}(i=1, \cdots, D)$ from the signature $g$, where $\delta_{1}>\cdots>\delta_{D}>0$. Let

$$
\mathcal{C}^{\varepsilon}(g)=\bigcup_{i=1}^{D} \mathcal{C}^{\delta_{i} ; D-i+1}
$$

be the totality of domains in each of them. $\mathcal{C}^{\varepsilon}(g)$ will be our single geometric scheme on the $\varepsilon$-scale in which we are going to construct the polygonal approximation. The notation emphasizes that it is determined by the signature $g$ as well as its dependence on $\varepsilon$. The previous Figure 5.3 also illustrates the construction of $\mathcal{C}^{\mathcal{E}}(g)$ when $D=2$.

Lemma 5.3. Let $A, B \in \mathcal{C}^{\varepsilon}(g)$, then $\bar{A} \cap \bar{B}=\emptyset$.

Proof. The case when $A, B$ come from the same geometric scheme $\mathcal{C}^{\delta_{i} ; D-i+1}$ is contained in Lemma 5.2. Now suppose that $A=C_{z}^{\delta_{i} ; D-i+1}$ and $B=C_{w}^{\delta_{j} ; D-j+1}$ for some $i<j$ with $z \in V_{D-i+1}$ and $w \in V_{D-j+1}$ respectively. By definition

This article is protected by copyright. All rights reserved. 
there exists some $I$ such that $z^{I}$ is an integer and $w^{I}$ is a half-integer. If $a \in \bar{A} \cap \bar{B}$, from the construction of our domains we know that

$$
\left|a^{I}-\varepsilon z^{I}\right| \leqslant \frac{\varepsilon-\delta_{i}}{2},\left|a^{I}-\varepsilon w^{I}\right| \leqslant \frac{\delta_{j-1}}{4} .
$$

But since $\delta_{j-1} \leqslant \delta_{i}$, we obtain that

$$
\left|z_{k}-w_{k}\right| \leqslant \frac{1}{2}-\frac{\delta_{i}}{4 \varepsilon}
$$

which is a contradiction. Therefore, $\bar{A} \cap \bar{B}=\emptyset$.

So far we have not seen how the stable quantities $\mathfrak{s}_{i}(i=1, \cdots, D)$ play a role in our reconstruction problem because the previous construction certainly applies to arbitrary stable quantities with respect to the corresponding geometric schemes. This will be clear in the next subsection.

\subsection{The Convergence}

Recall from Section 3 that $W_{0}$ is the space of continuous paths in $E_{\lfloor p\rfloor}$ which do not stay constant over any positive time period, and modulo reparametrization $\left(W_{0}^{\sim}, d\right)$ is a metric space. Moreover, the set of equivalence classes for treereduced weakly geometric $p$-rough paths is canonically embedded in $W_{0}^{\sim}$.

Let $\mathbf{X}$ be a simple tree-reduced weakly geometric $p$-rough path with signature $g$. Our goal is to reconstruct $\mathbf{X}$ modulo reparametrization, i.e. the equivalence class $[\mathbf{X}]$.

As before, in the geometric scheme $\mathcal{C}^{\varepsilon}(g)$, we define the word

$$
\mathbf{m}^{\varepsilon}=\left(m_{0}^{\varepsilon}=0, m_{1}^{\varepsilon}, \cdots, m_{L^{\varepsilon}}^{\varepsilon}\right)
$$

over the alphabet $V=V_{1} \cup \cdots \cup V_{D}$ corresponding to the ordered sequence of domains in $\mathcal{C}^{\varepsilon}$ visited by $\mathbf{X}$, which is again determined by the signature explicitly. We also define the corresponding entry times $\left\{\tau_{k}^{\varepsilon}: 0 \leqslant k \leqslant L^{\varepsilon}\right\}$, which is not determined by the signature as it is invariant under reparametrization.

Now we construct the associated polygonal approximation of $\mathbf{X}$ by joining the points in $\varepsilon \mathbf{m}^{\varepsilon}$ in order and parametrizing it according to the successive entry times $\tau_{k}^{\varepsilon}$. More precisely, we define

$$
\mathbf{X}_{t}^{\varepsilon}=\frac{\tau_{k}^{\varepsilon}-t}{\tau_{k}^{\varepsilon}-\tau_{k-1}^{\varepsilon}}\left(\mathbf{1}+\varepsilon m_{k-1}^{\varepsilon}\right)+\frac{t-\tau_{k-1}^{\varepsilon}}{\tau_{k}^{\varepsilon}-\tau_{k-1}^{\varepsilon}}\left(\mathbf{1}+\varepsilon m_{k}^{\varepsilon}\right)
$$

for $t \in\left[\tau_{k-1}^{\varepsilon}, \tau_{k}^{\varepsilon}\right]$ if $1 \leqslant k \leqslant L^{\varepsilon}$ and

$$
\mathbf{X}_{t}^{\varepsilon}=\mathbf{1}+\varepsilon m_{L^{\varepsilon}}^{\varepsilon}
$$

for $t \in\left[\tau_{L^{\varepsilon}}^{\varepsilon}, 1\right]$.

Apparently the path $\mathbf{X}^{\varepsilon}$ is not determined by the signature $g$. However, its equivalence class $\left[\mathbf{X}^{\varepsilon}\right]$, being regarded as an element in $W_{0}^{\sim}$, is reconstructed by $g$ since it is determined by the word $\mathbf{m}^{\varepsilon}$ only.

This article is protected by copyright. All rights reserved. 
Our reconstruction for the non-self-intersecting case will be finished by proving that $\left[\mathbf{X}^{\varepsilon}\right]$ converges to $[\mathbf{X}]$ in $\left(W_{0}^{\sim}, d\right)$ as $\varepsilon \rightarrow 0$. This is based on the following crucial fact.

Proposition 5.2. Let $T^{\varepsilon}=\left(\bigcup_{C \in \mathcal{C}^{\varepsilon}} C\right)^{c}$ be the set of tunnels for the geometric scheme $\mathcal{C}^{\varepsilon}$. Then there do not exist $s<t$ such that

$$
\left|\mathbf{X}_{t}-\mathbf{X}_{s}\right| \geqslant 33 D^{\frac{3}{2}} \varepsilon
$$

and $\operatorname{Im}\left(\left.\mathbf{X}\right|_{[s, t]}\right) \subset T^{\varepsilon}$.

Proof. Assume on the contrary that such $s, t$ exist. Since $\operatorname{Im}\left(\left.\mathbf{X}\right|_{[s, t]}\right) \subset T^{\varepsilon}$, we know that $\mathbf{X}$ does not visit any domain in $\mathcal{C}^{\varepsilon}(g)$ during $[s, t]$. For $1 \leqslant i \leqslant D$, let $C_{i}$ be the last domain in $\mathcal{C}^{\delta_{i} ; D-i+1}$ visited by $\mathbf{X}$ before $s$, and let $C_{i}^{\prime}$ be the first domain in $\mathcal{C}^{\delta_{i} ; D-i+1}$ visited by $\mathbf{X}$ after $t$. It might be possible that only one of them exists or even both do not exist. Let $z_{i}, z_{i}^{\prime}$ be their centers respectively. Since there are at most $2 D$ of them, there exists some $1 \leqslant k \leqslant 3 D$, such that

$$
\mathbf{1}+\varepsilon z_{i}, \mathbf{1}+\varepsilon z_{i}^{\prime} \notin A_{11(k-1) \sqrt{D} \varepsilon, 11 k \sqrt{D} \varepsilon}\left(\mathbf{X}_{s}\right)
$$

for all $1 \leqslant i \leqslant D$, where

$$
A_{r, R}(a):=\left\{b \in E_{\lfloor p\rfloor}: r<|b-a|<R\right\}
$$

denotes the open $(r, R)$-annulus around $a$.

By continuity and (5.7), there exist $s_{1}<t_{1} \in[s, t]$ such that

$$
\left|\mathbf{X}_{s_{1}}-\mathbf{X}_{s}\right|=11(k-1) \sqrt{D} \varepsilon
$$

and

$$
\left|\mathbf{X}_{t_{1}}-\mathbf{X}_{s}\right|=11 k \sqrt{D} \varepsilon
$$

Let

$$
t_{2}=\inf \left\{u \in\left[s_{1}, t_{1}\right]:\left|\mathbf{X}_{u}-\mathbf{X}_{s}\right| \geqslant(11 k-5) \sqrt{D} \varepsilon\right\}
$$

and

$$
s_{2}=\sup \left\{u \in\left[s_{1}, t_{2}\right]:\left|\mathbf{X}_{u}-\mathbf{X}_{s}\right| \leqslant(11 k-6) \sqrt{D} \varepsilon\right\} .
$$

It follows that

$$
\left|\mathbf{X}_{t_{2}}-\mathbf{X}_{s_{2}}\right| \geqslant \sqrt{D} \varepsilon
$$

and $\operatorname{Im}\left(\left.\mathbf{X}\right|_{\left[s_{2}, t_{2}\right]}\right) \subset \bar{A}_{(11 k-6) \sqrt{D} \varepsilon,(11 k-5) \sqrt{D} \varepsilon}$ (the closed annulus).

Now assume that there exists some $u \in\left[s_{2}, t_{2}\right]$ with $\mathbf{X}_{u} \in \mathbf{1}+\varepsilon K_{D}$ (recall that $K_{D}$ is the open $D$-skeleton). It follows that there exists some $\delta<\delta_{1}$ such that $\mathbf{X}_{u} \in \mathbf{1}+H_{z}^{\delta ; D}$ for some $z \in V_{D}$. From the construction of $s_{2}, t_{2}$, we have

$$
\left|z-z_{1}\right|,\left|z-z_{1}^{\prime}\right| \geqslant 4 \sqrt{D}
$$

Since $\operatorname{Im}\left(\left.\mathbf{X}\right|_{[s, t]}\right) \subset T^{\varepsilon}$, we conclude that $\mathbf{m}^{\delta_{1} ; D}$ must be a proper subword of $\mathbf{m}^{\delta ; D}$. More precisely, if we write $\mathbf{m}^{\delta_{1} ; D}$ in the form

$$
\mathbf{m}^{\delta_{1} ; D}=\left(\mathbf{z}_{1}, z_{1}, z_{1}^{\prime}, \mathbf{z}_{1}^{\prime}\right),
$$

This article is protected by copyright. All rights reserved. 
where $\mathbf{z}_{1}, \mathbf{z}_{1}^{\prime}$ are the sections of $\mathbf{m}^{\delta_{1} ; D}$ before $z_{1}$ and after $z_{1}^{\prime}$ respectively, then the word

$$
\mathbf{w}=\left(\mathbf{z}_{1}, z_{1}, z, z_{1}^{\prime}, \mathbf{z}_{1}^{\prime}\right) \in \mathcal{W}^{D}
$$

is a subword of $\mathbf{m}^{\delta ; D}$. Therefore, by definition we have $\mathfrak{s}_{D}(\mathbf{w}) \leqslant \mathfrak{s}_{D}\left(\mathbf{m}^{\delta ; D}\right)$.

On the other hand, let $c$ be a maximal admissible chain in $\mathbf{m}^{\delta_{1} ; D}$, and let $m_{1}, m_{1}^{\prime}$ be the last and first letters in $c \cap\left(\mathbf{z}_{1}, z_{1}\right)$ and $c \cap\left(z_{1}^{\prime}, \mathbf{z}_{1}^{\prime}\right)$ respectively. If $\left|z_{1}-m_{1}\right|,\left|z_{1}^{\prime}-m_{1}^{\prime}\right| \leqslant 2 \sqrt{D}$, from (5.9) we know that

$$
\left|z-m_{1}\right|,\left|z-m_{1}^{\prime}\right| \geqslant 2 \sqrt{D},
$$

which implies that $\left(c \cap\left(\mathbf{z}_{1}, z_{1}\right), z, c \cap\left(z_{1}^{\prime}, \mathbf{z}_{1}^{\prime}\right)\right)$ is an admissible chain in $\mathbf{w}$, and thus

$$
\mathfrak{s}_{D}(\mathbf{w}) \geqslant \mathfrak{s}_{D}\left(\mathbf{m}^{\delta_{1} ; D}\right)+1 .
$$

Similarly, if $\left|z_{1}-m_{1}\right| \leqslant 2 \sqrt{D},\left|z_{1}^{\prime}-m_{1}^{\prime}\right| \geqslant 2 \sqrt{D}$ or if $\left|z_{1}-m_{1}\right| \geqslant 2 \sqrt{D}$, $\left|z_{1}^{\prime}-m_{1}^{\prime}\right| \leqslant 2 \sqrt{D}$, we have

$$
\mathfrak{s}_{D}(\mathbf{w}) \geqslant \mathfrak{s}_{D}\left(\mathbf{m}^{\delta_{1} ; D}\right)+2 .
$$

And if $\left|z_{1}-m_{1}\right|,\left|z_{1}^{\prime}-m_{1}^{\prime}\right| \geqslant 2 \sqrt{D}$, we have

$$
\mathfrak{s}_{D}(\mathbf{w}) \geqslant \mathfrak{s}_{D}\left(\mathbf{m}^{\delta_{1} ; D}\right)+3 .
$$

In other words, we conclude that $\mathfrak{s}_{D}\left(\mathbf{m}^{\delta_{1} ; D}\right)<\mathfrak{s}_{D}(\mathbf{w}) \leqslant \mathfrak{s}_{D}\left(\mathbf{m}^{\delta ; D}\right)$. But this contradicts the construction of $\delta_{1}$. Therefore, $\operatorname{Im}\left(\left.\mathbf{X}\right|_{\left[s_{2}, t_{2}\right]}\right) \subset \mathbf{1}+\varepsilon C_{D-1}$ (recall that $C_{D-1}$ is the closed $(D-1)$-skeleton).

Based on the construction of $\delta_{2}$, the same argument shows that $\operatorname{Im}\left(\left.\mathbf{X}\right|_{\left[s_{2}, t_{2}\right]}\right) \subset$ $\mathbf{1}+\varepsilon C_{D-2}$. Recursively, we conclude that $\operatorname{Im}\left(\left.\mathbf{X}\right|_{\left[s_{2}, t_{2}\right]}\right) \subset \mathbf{1}+\varepsilon C_{0}$. Note that in each step $i$ of the recursive argument, we should also consider the cases when one of $z_{i}, z_{i}^{\prime}$ does not exist and both of them do not exist, but these two cases are apparently simpler than the general discussion before.

Finally, since $C_{0}$ is a discrete space, from continuity we have $\left.\mathbf{X}\right|_{\left[s_{2}, t_{2}\right]}=$ const, which contradicts (5.8).

Now the proof is complete.

Finally, we are in a position to prove the following convergence result.

Theorem 5.1. For every $\varepsilon>0$, we have

$$
\sup _{t \in[0,1]}\left|\mathbf{X}_{t}^{\varepsilon}-\mathbf{X}_{t}\right| \leqslant 68 D^{\frac{3}{2}} \varepsilon .
$$

In particular,

$$
\lim _{\varepsilon \rightarrow 0}\left[\mathbf{X}^{\varepsilon}\right]=[\mathbf{X}] \text { in }\left(W_{0}^{\sim}, d\right) .
$$

Proof. Consider the time interval $\left[\tau_{k-1}^{\varepsilon}, \tau_{k}^{\varepsilon}\right]$. Let $C_{k-1}^{\varepsilon}, C_{k}^{\varepsilon}$ be the domain in $\mathcal{C}^{\varepsilon}(g)$ corresponding to the entry times $\tau_{k-1}^{\varepsilon}, \tau_{k}^{\varepsilon}$ respectively. Define

$$
t^{*}=\sup \left\{t \in\left[\tau_{k-1}^{\varepsilon}, \tau_{k}^{\varepsilon}\right]: \mathbf{X}_{u} \in C_{k-1}^{\varepsilon}\right\} .
$$

This article is protected by copyright. All rights reserved. 
If $\left|m_{k}^{\varepsilon}-m_{k-1}^{\varepsilon}\right|>34 D^{3 / 2}$, then we have

$$
\left|\mathbf{X}_{t^{*}}-\mathbf{X}_{\tau_{k}^{\varepsilon}}\right| \geqslant 34 D^{\frac{3}{2}} \varepsilon-2 \times \frac{\sqrt{D}}{2} \varepsilon \geqslant 33 D^{\frac{3}{2}} \varepsilon .
$$

Moreover, we know that $\operatorname{Im}\left(\mathbf{X}\left(\left.\right|_{\left[t^{*}, \tau_{k}^{\varepsilon}\right]}\right) \subset T^{\varepsilon}\right.$. This contradicts Proposition 5.2. Therefore,

$$
\left|m_{k}^{\varepsilon}-m_{k-1}^{\varepsilon}\right| \leqslant 34 D^{3 / 2} \text {. }
$$

Let $t \in\left[\tau_{k-1}^{\varepsilon}, \tau_{k}^{\varepsilon}\right]$. If $\left|\mathbf{X}_{t}-\left(\mathbf{1}+\varepsilon m_{k-1}^{\varepsilon}\right)\right|>68 D^{3 / 2} \varepsilon$, from (5.11) we know that

$$
\left|\mathbf{X}_{t}-\left(1+\varepsilon m_{k}^{\varepsilon}\right)\right| \geqslant 34 D^{\frac{3}{2}} \varepsilon .
$$

Therefore,

$$
\bar{B}\left(\mathbf{X}_{t}, 33 D^{\frac{3}{2}} \varepsilon\right) \bigcap\left(\overline{C_{k-1}^{\varepsilon}} \bigcup \overline{C_{k}^{\varepsilon}}\right)=\emptyset
$$

Define

$$
t^{\prime}=\inf \left\{t \in\left[t, \tau_{k}^{\varepsilon}\right]: \mathbf{X}_{t} \in \partial \bar{B}\left(\mathbf{X}_{t}, 33 D^{\frac{3}{2}} \varepsilon\right)\right\} .
$$

It follows that $\left|\mathbf{X}_{t^{\prime}}-\mathbf{X}_{t}\right|=33 D^{3 / 2} \varepsilon$ and $\operatorname{Im}\left(\left.\mathbf{X}\right|_{\left[t, t^{\prime}\right]}\right) \subset T^{\varepsilon}$. This is again a contradiction to Proposition 5.2. Therefore,

$$
\left|\mathbf{X}_{t}-\left(\mathbf{1}+\varepsilon m_{k-1}^{\varepsilon}\right)\right| \leqslant 68 D^{\frac{3}{2}} \varepsilon .
$$

The same argument shows that

$$
\left|\mathbf{X}_{t}-\left(\mathbf{1}+\varepsilon m_{k}^{\varepsilon}\right)\right| \leqslant 68 D^{\frac{3}{2}} \varepsilon .
$$

Finally, from the construction of $\mathbf{X}^{\varepsilon}$, we obtain that

$$
\sup _{t \in\left[\tau_{k-1}^{\varepsilon}, \tau_{k}^{\varepsilon}\right]}\left|\mathbf{X}_{t}^{\varepsilon}-\mathbf{X}_{t}\right| \leqslant 68 D^{\frac{3}{2}} \varepsilon .
$$

The same argument shows that

$$
\sup _{t \in\left[\tau_{L^{\varepsilon}}^{\varepsilon}, 1\right]}\left|\mathbf{X}_{t}^{\varepsilon}-\mathbf{X}_{t}\right| \leqslant 34 D^{\frac{3}{2}} \varepsilon .
$$

Therefore, (5.10) holds.

Remark 5.5. From a technical point one might observe that $\mathbf{X}^{\varepsilon}$ is actually not an element of $W_{0}$ since it stays constant on $\left[\tau_{L^{\varepsilon}}^{\varepsilon}, 1\right]$. However, we can modify $\mathbf{X}^{\varepsilon}$ over $\left[\tau_{L^{\varepsilon}}^{\varepsilon}, 1\right]$ to be non-constant in the $\varepsilon$-scale in any arbitrary way, so that the result of Theorem 5.1 is still valid.

This article is protected by copyright. All rights reserved. 


\section{The Reconstruction: General Case}

Now we turn to the general case. Let $\mathbf{X}$ be a tree-reduced weakly geometric $p$-rough path with signature $g$.

For each $N \geqslant\lfloor p\rfloor$, the truncated signature path of $\mathbf{X}$ up to degree $N$ is denoted by $X^{(N)}$. If $I$ is a word over $\{1, \cdots, d\}$ with $|I| \leqslant N, X_{t}^{I}$ denotes the $I$-th component of $X_{t}^{(N)}$.

The main ingredient here is to construct a degree $N(g) \geqslant\lfloor p\rfloor$ from the signature $g$, so that we can conclude: $\left|X_{t}^{I}\right| \leqslant 1 / 2$ for all words $I$ with $|I|>N(g)$ and all $t \in[0,1]$. Starting from this, the construction in the last section carries through on the Euclidean space $E_{N(g)}$ without much difficulty.

Now let $0<\delta<1 / 4$ be a parameter. Again $\delta$ should be regarded as a discrete parameter, and only the direction $\delta \rightarrow 0$ matters.

For each $N \geqslant\lfloor p\rfloor$, let $D_{N}$ be the dimension of the Euclidean space $E_{N}$. Define $A_{0}^{N}$ to be the set of points $z=\left(z^{I}\right)_{0 \leqslant|I| \leqslant N} \in E_{N}$ such that at least one of the components of $z$ is $\pm 1 / 2$ and the rest are of the form $z^{I}=n / 2$ where $n \in \mathbb{Z}$. Let $A^{N}=\{0\} \cup A_{0}^{N}$.

Given $z \in A^{N}$, let

$Q_{z}^{\delta ; N}=\left\{a \in E_{N}:\left|a^{I}-z^{I}\right|<\frac{1}{2}-\delta\right.$ if $z^{I} \in \mathbb{Z}$ and $\left|a^{I}-z^{I}\right|<\frac{\delta}{2}$ otherwise $\}$.

Let $\mathcal{Q}^{\delta ; N}$ be the geometric scheme on $E_{N}$ consisting of all the cubes $\mathbf{1}+Q_{z}^{\delta ; N}$ $\left(z \in A^{N}\right)$. Apparently the closure of these cubes are mutually disjoint. A crucial feature of $\mathcal{Q}^{\delta ; N}$ for us is the following:

$$
\left\{a \in E_{N}:\left|a^{I}\right|=\frac{1}{2} \text { for some } I\right\} \subset \bigcup_{0<\delta<\frac{1}{4}} \bigcup_{z \in A_{0}^{N}} Q_{z}^{\delta ; N} .
$$

Figure 6.1 illustrates the construction of $\mathcal{Q}^{\delta ; N}$ when $D_{N}=2$.

Define $L^{\delta ; N}$ and the word

$$
\mathbf{m}^{\delta ; N}=\left(m_{0}^{\delta ; N}=0, m_{1}^{\delta ; N}, \cdots, m_{L^{\delta ; N}}^{\delta ; N}\right)
$$

corresponding to the ordered sequence of cubes in $\mathcal{Q}^{\delta ; N}$ visited by the truncated signature path $X^{(N)}$ as before.

As in Section 5.1, for $l \geqslant 0$, let $\widetilde{\mathcal{W}}_{l}^{N}$ be the set of words $\mathbf{n}=\left(n_{0}=0, n_{1}, \cdots n_{l}\right)$ over the alphabet $A^{N}$ such that $n_{k} \neq n_{k-1}$ for $1 \leqslant k \leqslant l$. After pre-specifying a countable family of generating one forms over the geometric scheme $\mathcal{Q}^{\delta ; N}$ on $E_{N}$, we define $\chi(g ; l, \mathbf{n})$ in the same way as $(5.2)$, and also define

$$
l^{\delta ; N}=\sup \left\{l \geqslant 0: \chi(g ; l, \mathbf{n})=1 \text { for some } \mathbf{n} \in \widetilde{\mathcal{W}}_{l}^{N}\right\},
$$

where we set $l^{\delta ; N}=0$ if such $\mathbf{n}$ does not exist for every $l$. According to Proposition 5.1 (2), we know that $l^{\delta ; N}<\infty$. Let $\mathbf{n}^{\delta ; N}$ be a word in $\widetilde{\mathcal{W}}_{l^{\delta ; N}}^{N}$ such that $\chi\left(g ; l^{\delta ; N}, \mathbf{n}^{\delta ; N}\right)=1$. Again $l^{\delta ; N}$ and $\mathbf{n}^{\delta ; N}$ are determined by the signature $g$ explicitly.

This article is protected by copyright. All rights reserved. 


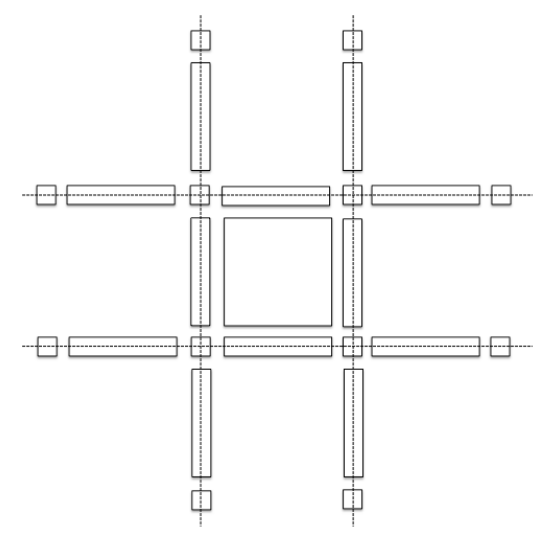

Figure 6.1: This figure illustrates the construction of $\mathcal{Q}^{\delta ; N}$ when $D_{N}=2$.

Now the key observation is the following: due to the decay of the homogeneous signature path of high degree, when $N$ becomes large, the high dimensional component of every letter in $\mathbf{n}^{\delta ; N}$ becomes zero. To be more precise, given $N<N^{\prime}$, let

$$
\pi_{N}^{N^{\prime}}: E_{N^{\prime}} \rightarrow \bigoplus_{i=N+1}^{N^{\prime}}\left(\mathbb{R}^{d}\right)^{\otimes i}
$$

be the projection onto the component of degree higher than $N$. If $\mathbf{n}$ is a word with letters in $E_{N^{\prime}}, \pi_{N}^{N^{\prime}}(\mathbf{n})$ is the word obtained by projecting every letter in $\mathbf{n}$ accordingly. Then we have the following result.

Lemma 6.1. There exists some $N_{0} \geqslant\lfloor p\rfloor$ independent of $\delta$, such that for any $N>N_{0}$ and $0<\delta<1 / 4$, we have

$$
\pi_{N_{0}}^{N}\left(\mathbf{n}^{\delta ; N}\right)=(0, \cdots, 0) .
$$

Proof. According to Lyons' extension theorem (Theorem 2.1), there exists some $N_{0} \geqslant\lfloor p\rfloor$ depending on the path $\mathbf{X}$, such that $\left|X_{t}^{I}\right| \leqslant 1 / 4$ for all $I$ with $|I|>N_{0}$ and all $t \in[0,1]$. If there exist $N>N_{0}$ and $0<\delta<1 / 4$ such that $\pi_{N_{0}}^{N}\left(\mathbf{n}^{\delta ; N}\right) \neq 0$, then there exists a letter $z \in \mathbf{n}^{\delta ; N}$ such that $z^{I} \neq 0$ for some $I$ with $N_{0}<|I| \leqslant$ $N$. From the construction of $\mathbf{n}^{\delta ; N}$, we conclude that the truncated signature path $X^{(N)}$ has visited the cube $Q_{z}^{\delta ; N}$ (for this it is helpful to see [3], Lemma 4.2). Therefore, there exists some $t \in[0,1]$ such that

$$
\left|X_{t}^{I}\right| \geqslant\left|z^{I}\right|-\left|X_{t}^{I}-z^{I}\right| \geqslant \begin{cases}1-\left(\frac{1}{2}-\delta\right)>\frac{1}{4}, & \text { if } z^{I} \text { is an integer; } \\ \frac{1}{2}-\frac{\delta}{2}>\frac{1}{4}, & \text { if } z^{I} \text { is a half-integer, }\end{cases}
$$

which is a contradiction. 


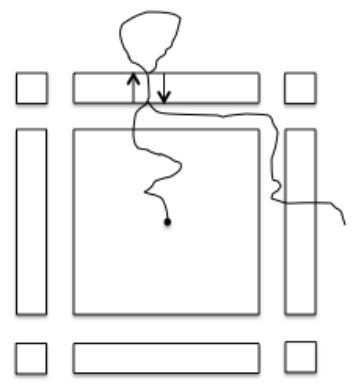

Figure 6.2: This figure illustrates the possibility that $\mathbf{n}^{\delta ; N}$ is a proper subword of $\mathbf{m}^{\delta ; N}$ when $D_{N}=2$. Here the underlying path is apparently tree-reduced. However, $\mathbf{n}^{\delta ; N}=((0,0),(1 / 2,0))$ and $\mathbf{m}^{\delta ; N}=((0,0),(0,1 / 2),(1 / 2,0))$.

Here a big difference from the non-self-intersecting case is that $l^{\delta ; N}$ can be strictly less than $L^{\delta ; N}$, and $\mathbf{n}^{\delta ; N}$ can just be some proper subword of $\mathbf{m}^{\delta ; N}$. Figure 6.2 illustrates this possibility when $D_{N}=2$. In this example, the reason is that any one form supported on the top long box integrates to zero as the underlying path cancels itself exactly inside this box. However, from Proposition 5.1 we know that if $l^{\delta ; N}=L^{\delta ; N}$, then $\mathbf{n}^{\delta ; N}$ must coincide with $\mathbf{m}^{\delta ; N}$. This is an important fact.

Let $N_{0}$ be given by Lemma 6.1. Given $N<N^{\prime}$, let $l_{N}^{N^{\prime}}$ be the lifting map

$$
\begin{aligned}
l_{N}^{N^{\prime}}: E_{N} & \rightarrow E_{N^{\prime}}=E_{N} \bigoplus \bigoplus_{i=N+1}^{N^{\prime}}\left(\mathbb{R}^{d}\right)^{\otimes i}, \\
z & \mapsto(z, 0) .
\end{aligned}
$$

If $\mathbf{n}$ is a word with letters in $E_{N}, l_{N}^{N^{\prime}}(\mathbf{n})$ is the word obtained by lifting every letter in $\mathbf{n}$ accordingly. The following result is important for us. It is where the tree-reduced property comes in.

Lemma 6.2. For $N^{\prime}>N \geqslant N_{0}$, we have

$$
\mathbf{m}^{\delta ; N^{\prime}}=l_{N}^{N^{\prime}}\left(\mathbf{m}^{\delta ; N}\right)
$$

for all $0<\delta<1 / 4$. In other words, knowing the discrete route of $X^{\left(N^{\prime}\right)}$ in the geometric scheme $\mathcal{Q}^{\delta ; N^{\prime}}$ is equivalent to knowing the one of $X^{(N)}$ in $\mathcal{Q}^{\delta ; N}$. Moreover, for each given $\delta$, when $N$ is large enough we have $\mathbf{n}^{\delta ; N}=\mathbf{m}^{\delta ; N}$.

Proof. Recall from the proof of Lemma 6.1 that $\left|X_{t}^{I}\right| \leqslant 1 / 4$ for $|I|>N_{0}$ and $t \in[0,1]$. Therefore, if $X_{t}^{\left(N^{\prime}\right)} \in Q_{z}^{\delta ; N^{\prime}}$ for some $z \in A^{N^{\prime}}$, then for any $I$ with $N<|I| \leqslant N^{\prime}$,

$$
\left|z^{I}\right| \leqslant\left|X_{t}^{I}\right|+\left|X_{t}^{I}-z^{I}\right| \leqslant \begin{cases}\frac{1}{4}+\frac{1}{2}-\delta, & \text { if } z^{I} \text { is an integer; } \\ \frac{1}{4}+\frac{\delta}{2}, & \text { if } z^{I} \text { is a half-integer }\end{cases}
$$

This article is protected by copyright. All rights reserved. 
It follows that $z^{I}=0$. Moreover, given $z \in A^{N}$, we have $X_{t}^{(N)} \in Q_{z}^{\delta ; N}$ if and only if $X_{t}^{\left(N^{\prime}\right)} \in Q_{l_{N}^{N^{\prime}}(z)}^{\delta ; N^{\prime}}$ since

$$
\left|X_{t}^{I}\right|=\left|X_{t}^{I}-0\right| \leqslant \frac{1}{4}<\frac{1}{2}-\delta
$$

for every $N<|I| \leqslant N^{\prime}$.

On the other hand, for given $\delta$, from the previous discussion we know that $\mathbf{m}^{\delta ; N}=l_{N_{0}}^{N}\left(\mathbf{m}^{\delta ; N_{0}}\right)$ for any $N>N_{0}$. Define $\left\{\tau_{k}^{\delta ; N}: 0 \leqslant k \leqslant L^{\delta ; N}\right\}$ to be the corresponding entry times. It is then easy to see that $\tau_{k}^{\delta ; N}=\tau_{k}^{\delta ; N_{0}}$ for every $0 \leqslant k \leqslant L^{\delta ; N}=L^{\delta ; N_{0}}$. For each $k$ we choose $\tau_{k}^{\delta ; N_{0}}<s_{k}<s_{k}^{\prime}<t_{k}^{\prime}<t_{k}<\tau_{k+1}^{\delta ; N_{0}}$ such that

$$
\operatorname{Im}\left(\left.X^{\left(N_{0}\right)}\right|_{\left[s_{k}, t_{k}\right]}\right) \subset Q_{m_{k}^{\delta, N_{0}}}^{\delta ; N_{0}},
$$

where $\tau_{L^{\delta ; N_{0}+1}}^{\delta ; N_{0}}:=1$. Note that for $N>N_{0}$ we also have

$$
\operatorname{Im}\left(\left.X^{(N)}\right|_{\left[s_{k}, t_{k}\right]}\right) \subset Q_{l_{N_{0}}^{N}\left(m_{k}^{\delta ; N}\right)}^{\delta,}
$$

Now let

$$
\eta=\inf _{0 \leqslant k \leqslant L^{\delta ; N_{0}}}\left\{s_{k}^{\prime}-s_{k}, t_{k}^{\prime}-s_{k}^{\prime}, t_{k}-t_{k}^{\prime}\right\} .
$$

Since $\mathbf{X}$ is a tree-reduced weakly geometric $p$-rough path, by a compactness argument (c.f. [4], Lemma 19) we know that there exists some $N_{1}>N_{0}$, such that $X_{s}^{(N)} \neq X_{t}^{(N)}$ for any $(s, t) \in[0,1]$ with $|t-s| \geqslant \eta$ and any $N>$ $N_{1}$. Keeping this separation property in mind, by applying exactly the same argument as in the proof of Proposition 5.1, we know that for each $N>N_{1}$, the extended signature for $X^{(N)}$ along certain compactly supported $C^{\alpha}$-one forms over the word $\mathbf{m}^{\delta ; N}$ is nonzero. Therefore, together with Proposition 5.1 (2), we conclude that $l^{\delta ; N}=L^{\delta ; N}$ and $\mathbf{n}^{\delta ; N}=\mathbf{m}^{\delta ; N}$.

For each $\delta$, we define

$$
N(g ; \delta)=\inf \left\{N \geqslant\lfloor p\rfloor: \pi_{N}^{N^{\prime}}\left(\mathbf{n}^{\delta ; N^{\prime}}\right)=(0, \cdots, 0) \forall N^{\prime}>N\right\},
$$

and define

$$
N(g)=\sup _{0<\delta<\frac{1}{4}} N(g ; \delta) .
$$

From Lemma 6.1 we have $N(g) \leqslant N_{0}$. Note that $N(g)$ is reconstructed from the signature $g$. Such $N(g)$ will serve our purpose. Namely, we have the following result.

Proposition 6.1. For any $I$ with $|I|>N(g)$ and $t \in[0,1]$, we have $\left|X_{t}^{I}\right| \leqslant 1 / 2$.

Proof. Assume on the contrary that there exists some $I$ with $|I|>N(g)$ and some $t$ such that $\left|X_{t}^{I}\right|>1 / 2$. Let $N=|I| \vee N_{0}$. By continuity, there exists some $t^{\prime} \in(0, t)$ such that $\left|X_{t^{\prime}}^{I}\right|=1 / 2$. Without loss of generality we assume that

This article is protected by copyright. All rights reserved. 
$X_{t^{\prime}}^{I}=1 / 2$. According to (6.1), there exist some $0<\delta<1 / 4, z \in A_{0}^{N}$, such that $X_{t^{\prime}}^{(N)} \in Q_{z}^{\delta ; N}$. Therefore, $z$ is a letter in $\mathbf{m}^{\delta ; N}$. Moreover, we have

$$
\left|\frac{1}{2}-z^{I}\right| \leqslant \begin{cases}\frac{1}{2}-\delta, & \text { if } z^{I} \text { is an integer; } \\ \frac{\delta}{2}, & \text { if } z^{I} \text { is a half-integer, }\end{cases}
$$

which implies that $z^{I}$ must be $1 / 2$.

According to Lemma 6.2 , we know that $\mathbf{m}^{\delta ; N^{\prime}}=l_{N}^{N^{\prime}}\left(\mathbf{m}^{\delta ; N}\right)$ and $\mathbf{n}^{\delta ; N^{\prime}}=$ $\mathbf{m}^{\delta ; N^{\prime}}$ when $N^{\prime}$ is large enough. Therefore,

$$
\pi_{N(g)}^{N^{\prime}}\left(\mathbf{n}^{\delta ; N^{\prime}}\right)=\pi_{N(g)}^{N^{\prime}}\left(\mathbf{m}^{\delta ; N^{\prime}}\right) .
$$

But from the previous discussion we know that the right hand side of the above identity contains a component $z^{I}=1 / 2$, thus $\pi_{N(g)}^{N^{\prime}}\left(\mathbf{n}^{\delta ; N^{\prime}}\right)$ is nonzero. This contradicts the definition of $N(g)$.

The final step is to develop exactly the same construction as in the non-selfintersecting case over the Euclidean space $E_{N(g)}$.

The only additional point is to see how to reconstruct the discrete route of $X^{(N(g))}$ in a given geometric scheme in $E_{N(g)}$ from the signature $g$. Let $\mathcal{C}=\left\{C_{i}: i \in \mathcal{I}\right\}$ be a given geometric scheme and let $\mathbf{m}^{\mathcal{C}}$ be the word over the alphabet $\mathcal{I}$ corresponding to the ordered sequence of domains in $\mathcal{C}$ visited by $X^{(N(g))}$. As before, in general we cannot expect that $\mathbf{m}^{\mathcal{C}}$ can be reconstructed from $g$ by computing extended signatures in $E_{N(g)}$ only, and we need to lift the construction to higher degrees. For each $N>N(g)$, let $\mathcal{C}^{N}$ be the geometric scheme in $E_{N}$ defined by

$$
\mathcal{C}^{N}=\left\{C_{i} \times U_{N}: i \in \mathcal{I}\right\},
$$

where $U_{N}$ is the cube in $\oplus_{i=N(g)+1}^{N}\left(\mathbb{R}^{d}\right)^{\otimes i}$ given by

$$
U_{N}=\left\{\left(z^{I}\right)_{N(g)<|I| \leqslant N}:\left|z^{I}\right|<1 \text { for every } I\right\} .
$$

From Proposition 6.1, we know that visiting $C_{i}$ by $X^{(N(g))}$ is equivalent to visiting $C_{i} \times U_{N}$ by $X^{(N)}$, i.e. $\mathbf{m}^{\mathcal{C}}=\mathbf{m}^{\mathcal{C} ; N}$. Moreover, the same argument as in the proof of Lemma 6.2 shows that $\mathbf{n}^{\mathcal{C} ; N}=\mathbf{m}^{\mathcal{C} ; N}$ when $N$ is large enough, where $\mathbf{n}^{\mathcal{C} ; N}$ is defined in the same way as $\mathbf{n}^{\delta ; N}$ before by computing extended signatures in $E_{N}$. Therefore, the word $\mathbf{n}^{\mathcal{C} ; N}$ is stable as $N \rightarrow \infty$ (here the alphabet is always $\mathcal{I}$ for every $N$ ), and we obtain that

$$
\mathbf{m}^{\mathcal{C}}=\lim _{N \rightarrow \infty} \mathbf{n}^{\mathcal{C} ; N}=\mathbf{n}^{\mathcal{C} ; N_{1}(g)},
$$

where

$$
N_{1}(g)=\inf \left\{N>N(g): \mathbf{n}^{\mathcal{C} ; N^{\prime}}=\mathbf{n}^{\mathcal{C} ; N} \forall N^{\prime}>N\right\}<\infty .
$$

In particular, $\mathbf{m}^{\mathcal{C}}$ is determined by the signature $g$ explicitly.

Finally, if we look back into the discussion in Section 5.2 and 5.3 , once $\mathbf{m}^{\mathcal{C}}$ is known for any geometric scheme $\mathcal{C}$ we are concerned with, the whole argument 
relies only on continuity (not even the rough path nature of the underlying path). In particular, by applying the same construction of geometric schemes as in the non-self-intersecting case, we obtain an approximating sequence $X^{\varepsilon}$ of polygonal paths for the truncated signature path $X^{(N(g))}$ on $E_{N(g)}$. Let $\mathbf{X}^{\varepsilon}$ be the projection of $X^{\varepsilon}$ onto $E_{\lfloor p\rfloor}$ and let $\left[\mathbf{X}^{\varepsilon}\right]$ be the corresponding equivalence class in $\left(W_{0}^{\sim}, d\right)$. Then we have the following convergence result. Remark 5.5 applies in the same way here.

Theorem 6.1. For every $\varepsilon>0$, we have

$$
\sup _{t \in[0,1]}\left|X_{t}^{\varepsilon}-X_{t}^{(N(g))}\right|_{E_{N(g)}} \leqslant 68 D_{N(g)}^{\frac{3}{2}} \varepsilon .
$$

In particular,

$$
\lim _{\varepsilon \rightarrow 0}\left[\mathbf{X}^{\varepsilon}\right]=[\mathbf{X}] \text { in }\left(W_{0}^{\sim}, d\right) .
$$

It is worthwhile to point out that a direct consequence of Theorem 6.1 is the following uniqueness result for the signature of a rough path.

Corollary 6.1. Let $\mathbf{X}, \mathbf{Y}$ be two tree-reduced weakly geometric p-rough paths in the sense that their signature paths $\mathbb{X}, \mathbb{Y}$ are both simple. Then $\mathbb{X}$ and $\mathbb{Y}$ are equal up to reparametrization if and only if $S(\mathbf{X})_{0,1}=S(\mathbf{Y})_{0,1}$. In particular, in this case $\mathbf{X}$ and $\mathbf{Y}$ are equal up to reparametrization.

Proof. Suppose that $S(\mathbf{X})_{0,1}=S(\mathbf{Y})_{0,1}=g$. It suffices to show that $\mathbb{X}$ and $\mathbb{Y}$ have the same image.

From the proof of Theorem 6.1, we know that for every $N>N(g)$ where $N(g)$ is defined by $(6.3)$, as a trajectory on $E_{N}, X^{(N)}$ (modulo reparametrization) can be reconstructed from $g$, and similarly for $Y^{(N)}$. Therefore, $X^{(N)}$ and $Y^{(N)}$ are equal up to reparametrization.

Now fix $t \in[0,1]$. It follows that for every $N>N(g)$, there exists $s_{N} \in[0,1]$ such that $X_{t}^{(N)}=Y_{s_{N}}^{(N)}$. From compactness we may assume without loss of generality that $s_{N} \rightarrow s \in[0,1]$ as $N \rightarrow \infty$. By projection we conclude that

$$
X_{t}^{(N)}=Y_{s_{N^{\prime}}}^{(N)}
$$

for every $N^{\prime}>N>N(g)$. By sending $N^{\prime} \rightarrow \infty$, we obtain that $X_{t}^{(N)}=Y_{s}^{(N)}$ for every given $N>N(g)$. This implies that

$$
\mathbb{X}_{t}=\mathbb{Y}_{s} \in \operatorname{Im}(\mathbb{Y})
$$

Remark 6.1. Corollary 6.1 does not cover the general uniqueness result in [4] (c.f. Theorem 2.3) as the signature is not able to detect any tree-like pieces of the underlying rough path. However, Corollary 6.1 is indeed the key ingredient in proving the general uniqueness result (the necessity part: trivial signature implies being tree-like) as it immediately leads to a canonical real tree structure on the signature group $\mathfrak{S}_{p}$. The underlying rough path is then realized as a continuous loop in the real tree $\mathfrak{S}_{p}$.

This article is protected by copyright. All rights reserved. 


\section{$7 \quad$ Final Remarks}

We give a few remarks in the following to conclude the present paper.

1. In summary, the general idea of our reconstruction consists of two parts: the signature determines the discrete route in any given geometric scheme, and the discrete route gives back our underlying path by refining the geometric scheme in a way determined by the signature. The first part is an easy consequence of the algebraic structure of signature, while the second part is the key ingredient of the present work. If we look back into the discussion, it is not hard to see that the development of the second part relies only on the continuity of our underlying path. In other words, if the discrete route of a continuous path is known for any given geometric scheme of the type in Section 5.1, our reconstruction gives back the original path. This works for all continuous paths without any regularity assumption.

The reason we cannot expect that the discrete route would yield the underlying path when the geometric scheme is fine enough and the second part is necessary is that there are positive gaps among the domains in our geometric scheme. Such construction is essential in our discussion. Removing the gaps ruins the whole argument as the discrete route is no longer well-defined. On the other hand, one might ask if we could use finitely many different geometric schemes to cover all the gaps so that our argument could be simplified. But it seems difficult to achieve because there is not a canonical way to embed two discrete routes arising from two different geometric schemes into a single word. Nevertheless, we expect that there might be some way to do this and our construction is certainly not the only possibility.

2. We expect that a recursive construction of geometric schemes in Section 5 is not necessary, and there might be a way to construct a single geometric scheme $\mathcal{C}^{\delta}$ in one go with one parameter $\delta$ only (recall that $\varepsilon$ is fixed). This requires a more difficult construction of a stable quantity as we cannot expect that every domain in $\mathcal{C}^{\delta}$ is expanding as $\delta \rightarrow 0$ (it is hard to see whether the quantity we have constructed in Section 5.2 is stable in this case).

3 . The reconstruction problem is essentially (countably) infinite dimensional. As we are looking for a universal way to treat every path equally, we should expect that all information of signature as an infinite dimensional object is to be used. From a practical side, one might wonder if in each $\varepsilon$-step, we could truncate our construction to a situation involving only finite information (depending on $\varepsilon$ ) and convergence still hold. We expect that this is possible although we do not pursue along this direction in our work and we are more focused on the theoretical side.

However, we want to emphasize that even with the possibility of proving convergence, we expect that any attempt to obtain a quantitative error estimate for a finite scheme reconstruction should involve an a priori control on the behavior of the class of paths we are considering (for instance, an a prior uniform regularity estimate or an a priori control on certain geometric property). More precisely, it is unlikely to expect the existence of a quantitative result of the

This article is protected by copyright. All rights reserved. 
following type:

$$
d\left(\xi^{\varepsilon}(g),[\mathbf{X}]\right) \leqslant C(\varepsilon, g), \quad \forall \varepsilon>0, g \in \mathfrak{S}_{p},
$$

where $\xi^{\varepsilon}(g) \in W_{0}^{\sim}$ is constructed by using finitely many components of $g$ through finitely many steps of computation, and $C(\varepsilon, g)$ is an explicit function depending only on $\varepsilon$ and $g$ such that

$$
\lim _{\varepsilon \rightarrow 0} C(\varepsilon, g)=0, \forall g \in \mathfrak{S}_{p} .
$$

This is the nature of the reconstruction problem. To expect an error estimate like (7.1) for a finite scheme reconstruction, we believe that the restriction to an a priori subset of $\mathfrak{S}_{p}$ is necessary. This leaves an interesting question from the computational point of view, for instance within our reconstruction method. But this is beyond the scope of the present paper.

\section{Acknowledgement}

The author wishes to thank Professor Terry Lyons for his valuable suggestions on the present work. The author is supported by the ERC grant (Grant Agreement No.291244 Esig).

\section{References}

[1] S. Aida, Vanishing of one-dimensional $L^{2}$-cohomologies of loop groups, $J$. Funct. Anal. 261 (8): 2164-2213, 2011.

[2] T. Bagby, L. Bos and N. Levenberg, Multivariate simultaneous approximation, Constr. Approx. 18 (3): 569-577, 2002.

[3] H. Boedihardjo and X. Geng, The uniqueness of signature problem in the non-Markov setting, to appear in Stochastic Process. Appl., 2015.

[4] H. Boedihardjo, X. Geng, T. Lyons and D. Yang, The Signature of a rough path: uniqueness, Adv. Math. 293: 720-737, 2016.

[5] T. Cass, B.K. Driver, N. Lim and C. Litterer, On the integration of weakly geometric rough paths, to appear in J. Math. Soc. Japan, 2015.

[6] T. Cass and P. Friz, Densities for rough differential equations under Hörmander's condition, Ann. of Math. 171 (3): 2115-2141, 2010.

[7] K.T. Chen, Iterated integrals and exponential homomorphisms, Proc. London Math. Soc. 4 (3): 502-512, 1954.

[8] K.T. Chen, Integration of paths, geometric invariants and a generalized Baker-Hausdorff formula, Ann. of Math. 65 (1): 163-178, 1957.

This article is protected by copyright. All rights reserved. 
[9] K.T. Chen, Integration of paths-a faithful representation of paths by noncommutative formal power series, Trans. Amer. Math. Soc. 89: 395-407, 1958.

[10] P. Friz and N. Victoir, Multidimensional stochastic processes as rough paths, Cambridge Studies of Advanced Mathematics, Vol. 120, Cambridge University Press, 2010.

[11] X. Geng and Z. Qian, On an inversion theorem for Stratonovich's signatures of multidimensional diffusion paths, to appear in Ann. Inst. Poincaré (B), 2015 .

[12] B.M. Hambly and T. Lyons, Uniqueness for the signature of a path of bounded variation and the reduced path group, Ann. of Math. 171 (1): 109-167, 2010.

[13] M. Ledoux, Z. Qian and T. Zhang, Large deviations and support theorem for diffusion processes via rough paths, Stochastic Process. Appl. 102 (2): 265-283, 2002.

[14] D. Levin, T. Lyons and $\mathrm{H}$. Ni, Learning from the past, predicting the statistics for the future, learning an evolving system, arXiv: 1309.0260, 2013.

[15] Y. Le Jan and Z. Qian, Stratonovich's signatures of Brownian motion determine Brownian sample paths, Probab. Theory Relat. Fields 157: 209-223, 2013.

[16] T. Lyons, Differential equations driven by rough signals, Rev. Mat. Iberoamericana 14 (2): 215-310, 1998.

[17] T. Lyons, H. Ni and H. Oberhauser, A feature set for streams and an application to high-frequency financial tick data, Proceedings of the 2014 International Conference on Big Data Science and Computing, ACM, 2014.

[18] T. Lyons and W. Xu, Hyperbolic development and inversion of signature, arXiv: 1507.00286, 2015.

[19] T. Lyons and W. Xu, Inverting the signature of a path, arXiv: 1406.7833, 2015.

[20] M. Hairer, A theory of regularity structures, Invent. Math. 198 (2): 269$504,2014$.

[21] C. Reutenauer, Free Lie algebras, Clarendon Press, 1993.

This article is protected by copyright. All rights reserved. 


\section{University Library}

\section{- M M I N E R VA A gateway to Melbourne's research publications}

Minerva Access is the Institutional Repository of The University of Melbourne

Author/s:

Geng, X

Title:

Reconstruction for the signature of a rough path

Date:

2017-03-01

Citation:

Geng, X. (2017). Reconstruction for the signature of a rough path. PROCEEDINGS OF THE LONDON MATHEMATICAL SOCIETY, 114 (3), pp.495-526. https://doi.org/10.1112/ plms.12013.

Persistent Link:

http://hdl.handle.net/11343/292326 